\title{
Evolution of protoplanetary disks: constraints from DM Tauri and GM Aurigae
}

\author{
R. Hueso ${ }^{1}$ and T. Guillot ${ }^{2}$ \\ ${ }^{1}$ Física Aplicada I, ETS Ing. Universidad del País Vasco, Alda. Urquijo s/n, 48013 Bilbao, Spain \\ e-mail: wubhualr@lg.ehu.es \\ 2 Laboratoire Cassiopée, CNRS UMR 6202, Observatoire de la Côte d'Azur, Nice, France \\ e-mail: guillot@obs-nice.fr
}

Received 26 August 2004 / Accepted 21 June 2005

\section{ABSTRACT}

We present a one-dimensional model of the formation and viscous evolution of protoplanetary disks. The formation of the early disk is modeled as the result of the gravitational collapse of an isothermal molecular cloud. The disk's viscous evolution is integrated according to two parameterizations of turbulence: the classical $\alpha$ representation and a $\beta$ parameterization, representative of non-linear turbulence driven by the keplerian shear. We apply the model to DM Tau and GM Aur, two classical T-Tauri stars with relatively well-characterized disks, retrieving the evolution of their surface density with time. We perform a systematic Monte-Carlo exploration of the parameter space (i.e. values of the $\alpha-\beta$ parameters, and of the temperature and rotation rate in the molecular cloud) to find the values that are compatible with the observed disk surface density distribution, star and disk mass, age and present accretion rate. We find that the observations for DM Tau require $0.001<\alpha<0.1$ or $2 \times 10^{-5}<\beta<5 \times 10^{-4}$. For GM Aur, we find that the turbulent viscosity is such that $4 \times 10^{-4}<\alpha<0.01$ or $2 \times 10^{-6}<\beta<8 \times 10^{-5}$. These relatively large values show that an efficient turbulent diffusion mechanism is present at distances larger than $\sim 10$ AU. This is to be compared to studies of the variations of accretion rates of T-Tauri stars versus age that mostly probe the inner disks, but also yield values of $\alpha \sim 0.01$. We show that the mechanism responsible for turbulent diffusion at large orbital distances most probably cannot be convection because of its suppression at low optical depths.

Key words. accretion, accretion disks - solar system: formation - planetary systems: formation planetary systems: protoplanetary disks

\section{Introduction}

The presence of circumstellar disks has been proposed long ago as a necessary preliminary of star formation, simply because the decrease of the moment of inertia of a collapsing molecular cloud core by a factor $10^{16}$ prevents the direct formation of a central compact object (the star) without a significant loss of angular momentum. These disks are now routinely discovered, and both statistical information of disk properties obtained from the spectral energy distributions and characterization of individual disks from direct measurements can be obtained (see Protostars $\mathcal{E}$ Planets $I V$ for detailed reviews on the subject).

The basic principle yielding the formation of a disk around a protostar also governs its evolution: material is allowed to be accreted from the disk onto the protostar only by decreasing its specific angular momentum. The conservation of mass and total angular momentum implies that some of the material in the disk must be transported outward in order for accretion to be possible (e.g. Lynden-Bell \& Pringle 1974; Pringle 1981; Cassen 1994).
One of the major puzzles in understanding circumstellar disks remains the mechanism by which angular momentum is transported. It is easy to show that viscosity on a microscopic scale is unable to explain the dissipation of disks on any reasonable timescale. Beyond that, the problem resides not in finding a mechanism of angular momentum transport but rather on deciding which one is appropriate and what are the magnitudes of the resulting angular momentum transport $\&$ associated heat dissipation (see e.g. Cassen 1994; Lin \& Papaloizou 1996; Stone et al. 2000). It is generally believed that disks of relatively small masses transport their angular momentum by turbulence and that their evolution can be calculated using an effective turbulent viscosity. The parameterization of this viscosity then allows the calculation of the evolution of circumstellar disks to be performed over the timescales of interest (Myr).

In this paper, we confront a 1-dimensional theoretical model of the formation and evolution of circumstellar disks to direct observations of the disks around the T-Tauri stars DM Tauri and GM Aurigae. These disks have the particularity that their outer surface densities (between $\sim 50$ and $800 \mathrm{AU}$ ) are constrained by millimeter-wavelengths 
observations (Guilloteau \& Dutrey 1998; Dutrey et al. 1998), continuum IR emission from the dust (Kitamura et al. 2002), and observations of star light scattered by the disk (Schneider et al. 2003). We use two different parameterizations of the turbulent viscosity (named $\alpha$ and $\beta$, see Sect. 3 hereafter) and two models of molecular cloud collapse.

The simplicity of our model allows for an extensive exploration of the space of parameters. In total more than 10000 models were run. We can therefore constrain the magnitude of the turbulent viscosity necessary to produce disks of the observed age, mass, and surface density. The observational constraints however are subject to very large uncertainties and instead of one simple scenario of formation and evolution for each disk we find several kind of solutions to the current state of DM Tau and GM Aur. Our results can be used to address the questions of the source of the turbulence, the initial conditions and the type of collapse of the molecular cloud. They are also helpful in providing a framework for planet formation, as all the important quantities such as densities and temperatures can be calculated as a function of time and distance to the central star.

The structure of this article is as follows: in Sect. 2 we present the observational characteristics of the DM Tau and GM Aur systems, and in particular the parameters that must be fitted by the models. The numerical model itself and the physics behind it (including the possible sources of angular momentum transport) are presented in Sect. 3. Section 4 presents some model examples and the strategy employed to fit the observations. We present our selected sets of models in Sect. 5 and discuss the consequences for the disks global evolution, the turbulent angular momentum transport and the initial type of collapse. Finally, Sect. 6 summarizes the main results of the article.

\section{Observations of circumstellar disks: DM Tau and GM Aur}

DM Tau and GM Aur are both located in the Taurus-Auriga region, a young stellar association relatively close to Earth, $\sim 140$ pc (Kenyon et al. 1994), with a low abundance of massive stars and a relatively cold $(\sim 10-20 \mathrm{~K})$ environment (van Dishoeck et al. 1993). These stars have been chosen for this study because their disks have been characterized at millimeter to ultraviolet wavelengths, and because the emission of $\mathrm{CO}$ and its isotomers has been detected by millimetric interferometers to large distances (500 to $800 \mathrm{AU}$ ) to the stars. For these disks, we thus have access to observations sensitive to the presence of dust (spectral energy distribution in the infrared and at millimetric wavelength, visible images of reflected stellar light, images in the millimetric continuum), to the presence of hot inner gas accreted by the star (excess emission mainly in the $U$ band) and to the presence of cold gaseous $\mathrm{CO}$ in the outer parts of the disks (interferometric ${ }^{12} \mathrm{CO}$ and ${ }^{13} \mathrm{CO}$ emission maps).

\subsection{DM Tau}

DM Tau is a low mass $\left(0.50 M_{\odot}\right)$ relatively old T-Tauri star $\left(\sim 5 \times 10^{6} \mathrm{yr}\right)$. Its gas disk was discovered by Guilloteau \& Dutrey (1994) with the IRAM 30-m telescope and independently by Handa et al. (1995). Further observations and a $\chi^{2}$ analysis (Guilloteau \& Dutrey 1998) allowed the determination of the disk inclination and showed a rotation rate consistent with a keplerian profile. More recently, Dartois et al. (2003) combined observations of CO lines of different Cisotopes to better infer the surface densities and also estimate the disk vertical temperature profile. Their study confirmed theoretical expectations that the disk mid-plane at $\sim 100 \mathrm{AU}$ is colder $(T \sim 15 \mathrm{~K})$ than $2-3$ scale heights higher $(T \sim 30 \mathrm{~K})$. The disk radius, $850 \mathrm{AU}$, and the slope of the density profile, $p=1.5$ derived by Guilloteau and Dutrey differ from a previous study by Saito et al. (1995) who derived $350 \mathrm{AU}$ and $p=2.0 \pm 0.3$. The discrepancy can probably be attributed to the lower resolution ( $5^{\prime \prime}$ compared to $\left.3^{\prime \prime}\right)$ and sensitivity of the observations by Saito et al.

The outer disk radius measured for DM Tau either from the mm continuum emission (Kitamura et al. 2002) or from HST near IR coronographic images (Grady et al. 2002) is assumed to be a lower limit because of the systematically smaller lower sensitivities (in terms of equivalent surface densities) of these techniques compared to the $\mathrm{CO}$ observations.

Recent observations of DM Tau in the near- to mid-infrared $(2.9-13.5 \mu \mathrm{m})$ and preliminary radiative transfer models by Bergin et al. (2004) seem to indicate that the inner region of the disk consists of three parts: (i) close to the star, and up to $\sim 4 \mathrm{AU}$, a flat, optically thin disk, cleared of most of its dust (and probably gas); (ii) At 4 AU, a "wall", heated by direct stellar irradiation to $\sim 120 \mathrm{~K}$, and extending vertically to $\pm 0.5 \mathrm{AU}$ relative to the midplane; (iii) A "standard" optically thick disk beyond that distance.

\subsection{GM Aurigae}

The evidence of the presence of a disk around GM Aur was first obtained from radiometric observations by Koerner et al. (1993). Higher resolution interferometric maps were later obtained by Dutrey et al. (1998), and Kitamura et al. (2002) using the same techniques as for DM Tau. Depending on the inclination of the disk, two solutions can be found for the star mass, i.e. $M_{*}=0.5$ or $0.9 M_{\odot}$ with most probable values close to 0.9 . Its disk appears to be slightly smaller than that of DM Tau, and have an outer radius $\sim 525 \mathrm{AU}$, as determined from ${ }^{12} \mathrm{CO}$ observations. High-resolution coronographic HST images of scattered near-infrared light (Wood et al. 2002; Schneider et al. 2003) indicate that the disk is outwardly flared and extends at least to $300 \mathrm{AU}$.

As for DM Tau, the near- to mid-infrared observations indicate the presence of an inner disk clearing within $6.5 \mathrm{AU}$, with a $120 \mathrm{~K}$ "wall" at that distance and an optically thick disk beyond (Bergin et al. 2004). The presence of such a gap (but a slightly smaller, i.e. 4 AU one) was also an outcome of the modeling of a less accurate SED (Chiang \& Goldreich 1999; Wood et al. 2002), and attributed to the presence of a $\sim 2 M_{\text {J }}$ planet orbiting at 2.5 AU (Wood et al. 2002; Rice et al. 2003). This explanation remains mostly speculative at this point, however. 
Table 1. Physical parameters for DM Tau and GM Aur: stellar parameters are from Simon et al. (2001), CO constraints from Dartois et al. (2003) and Dutrey et al. (1998), dust emission constraints from Kitamura et al. (2002) (with an additional $1 / 3$ to 3 uncertainty factor - see text) and accretion rates from Hartmann et al. (1998) and Gullbring et al. (1998).

\begin{tabular}{lll}
\hline \hline System: & DM Tau & GM Aur \\
\hline Distance: & $140 \mathrm{pc} \pm 10 \%$ & $140 \mathrm{pc} \pm 10 \%$ \\
Age: & $1.5-7 \mathrm{Myr}$ & $1-10 \mathrm{Myr}$ \\
Spectral type: & $\mathrm{M} 1$ & $\mathrm{~K} 7$ \\
Star mass: & $0.4-0.6 M_{\odot}$ & $0.5-1.0 M_{\odot}$ \\
Luminosity: & $0.25 L_{\odot}$ & $0.74 L_{\odot}$ \\
Temperature: & $3720 \mathrm{~K}$ & $4060 \mathrm{~K}$ \\
Star radius: & $0.0065 \mathrm{AU}$ & $0.0085 \mathrm{AU}$ \\
\hline
\end{tabular}

Constraints from $\mathrm{CO}$ emission lines:

$\begin{array}{lll}R_{\text {out }} & \sim 850 \pm 20 \mathrm{AU} & 525 \pm 20 \mathrm{AU} \\ \Sigma_{(100 \mathrm{AU})} & >0.05 \mathrm{~g} \mathrm{~cm}^{-2} & - \\ \Sigma_{\left(R_{\text {out }}\right)} & >1.6 \times 10^{-3} \mathrm{~g} \mathrm{~cm}^{-2} & 3.0 \times 10^{-3} \mathrm{~g} \mathrm{~cm}^{-2}\end{array}$

Constraints from continuum dust emission:

\begin{tabular}{|c|c|c|c|}
\hline$R_{\text {out }}$ & $>$ & $500 \mathrm{AU}$ & $280 \mathrm{AU}$ \\
\hline$\Sigma_{(100 \mathrm{AU})}$ & $<$ & $4.3 \mathrm{~g} \mathrm{~cm}^{-2}$ & $23.0 \mathrm{~g} \mathrm{~cm}^{-2}$ \\
\hline$\Sigma_{(100 \mathrm{AU})}$ & $>$ & $0.23 \mathrm{~g} \mathrm{~cm}^{-2}$ & $1.4 \mathrm{~g} \mathrm{~cm}^{-2}$ \\
\hline$\tilde{\beta}$ & & $0.48-1.03$ & $1.11-1.34$ \\
\hline
\end{tabular}

Accretion rate:

$\log \dot{M} \quad=\quad-7.95 \pm 0.54 \quad-8.02 \pm 0.54$

$\left[M_{\odot} \mathrm{yr}^{-1}\right]$

\subsection{Inferred constraints}

We detail hereafter the observational constraints that are directly relevant to the disk evolution calculations and that are summarized in Table 1 . We choose to adopt relatively conservative constraints. (More restrictive constraints may be obtained from SED fitting and direct analysis of the emission maps which is beyond the scope of the present article.)

Stellar masses: they are inferred from the keplerian rotation rates measured in ${ }^{12} \mathrm{CO}$ (Simon et al. 2001). The uncertainty on this parameter is essentially due to that of the distance given by Hipparcos to an accuracy $\sim 10 \%$. The inferred stellar masses depend also on the the disk inclination. Ages: this is a crucial, but unfortunately very uncertain parameter entering the models. The uncertainty on the stellar masses propagates into that on the inferred age. Taking that into account, and using evolution tracks from Baraffe et al. (1998, 1998), we derive for both stars a range of ages that covers ages published by different groups (e.g. Guilloteau \& Dutrey 1998; Hartmann et al. 1998).

Accretion rates: as discussed by Hartmann et al. (1998), an accretion luminosity $L_{\mathrm{acc}}$ can be derived from the measured excess emission observed in the $U$ band. It is then turned into an accretion rate following the relation:

$\dot{M}=\frac{1}{\gamma} \frac{R_{\star}}{G M_{\star}} L_{\mathrm{acc}}$, where $R_{\star}$ and $M_{\star}$ are the radius and mass of the star, respectively, and $\gamma$ is a factor that accounts for the distance from which the material free-falls onto the star. This parameter is expected to be $\sim 0.8$ due to the presence of a magnetic cavity inside $5 R_{\star}$. The uncertainties on $\dot{M}$ stem both from those linked to the determination of the bolometric accretion luminosity, and those related to the uncertain $\gamma$. Hartmann et al. estimate the uncertainty on $\dot{M}$ to be within a factor 3, with more optimistic error estimates leading to a factor $\sim 2$, given the likely presence of the magnetic cavity.

Surface densities from measured $\mathrm{CO}$ emission maps: in the case of DM Tau, measurements of ${ }^{12} \mathrm{CO},{ }^{13} \mathrm{CO}$ and $\mathrm{C}^{18} \mathrm{O}$ lines allow for a direct determination of the density profile of gaseous CO from orbital distances $\sim 100 \mathrm{AU}$ to the outer disk (Dartois et al. 2003). Interestingly, the outer radius of the disk is different depending on the molecular species that is considered, which is interpreted as selective photodissociation in the outer disk. ${ }^{12} \mathrm{CO}$ being the most abundant isotomer, it is also the most resistant to photodissociation and yields the largest radius (used for this work). Because CO furthermore tends to condense in these cold regions (e.g. Aikawa et al. 1999), the gas mass can only be thought as a lower limit to the outer disk mass. A comparison of $\mathrm{CO}$ and dust emission in DM Tau indeed yields a disk mass that is smaller by a factor $\sim 5$ when inferred from the $\mathrm{CO}$ measurement than when inferred from the dust (Dartois et al. 2003). This appears to be consistent with CO condensation, but may also be partially explained by a larger continuum opacity. The same conclusions hold for GM Aur, but the set of observations is more limited, because it is based on ${ }^{13} \mathrm{CO} J=1 \rightarrow 0$ (Dutrey et al. 1998) and ${ }^{12} \mathrm{CO} J=2 \rightarrow 1$ (Dutrey et al. 1998) emission. In Table 1, the minimum values of the surface density required to explain the $\mathrm{CO}$ emission are derived from Dartois et al. (2003) for DM Tau and Dutrey et al. (1998) for GM Aur. Conservatively, we assumed that all CO was in gaseous form and with a solar abundance to derive these minimum densities.

Surface densities from continuum emission maps: because continuum millimetric and sub-millimetric emissions are optically thin, their measurements inform us on the surface density and global masses of the disks. However, for a given flux, the surface density (or equivalently disk mass) derived is inversely proportionnal to an uncertain opacity coefficient $\kappa_{v}$. Following Beckwith et al. (1990), this opacity is often approximated by:

$\kappa_{v}=\kappa_{0}\left(\frac{v}{10^{12} \mathrm{~Hz}}\right)^{\tilde{\beta}}$,

where $\kappa_{0} \approx 0.1 \mathrm{~cm}^{2} \mathrm{~g}^{-1}$ and $\tilde{\beta}$ are parameters that depend on the grain composition and size (e.g. Beckwith et al. 1990). $\kappa_{0}$ is the opacity per total mass (gas+dust) and therefore depends on an assumed dust to gas ratio. The $\tilde{\beta}$ parameter can be obtained from the slope of the spectral energy distribution in the mm region (e.g. Beckwith et al. 1990; Chiang $\&$ Goldreich et al. 1997). Uncertainties on $\kappa_{0}$ are often neglected in the calculation of disk masses and surface densities although they are probably large (Beckwith et al. 1990; 
Pollack et al. 1994; Hartmann et al. 1998; D'Alessio et al. 2001).

In this work, we adopt the matches to the SEDs and $2 \mathrm{~mm}$ emission maps obtained by Kitamura et al. (2002) to constrain the surface density at $100 \mathrm{AU}$. We choose not to use their constraints on the disk masses and radii and on the slope of the surface density profile because the loss of sensitivity of the observations at large orbital distances and the limited spatial resolution imply that these are very model-dependant. Kitamura et al. do include a range of allowed $\tilde{\beta}$ values to estimate the characteristics of the disks, but do not allow for variations in $\kappa_{0}$. In pratice, the values of $\tilde{\beta}$ for DM Tau are smaller (by $\sim 0.45$ ) than those for GM Aur. Given the standard opacity law (Eq. (1)), this implies that the assumed $1.3 \mathrm{~mm}$ opacity is about twice larger for DM Tau than for GM Aur. On the other hand, models of grain growth indicate that low values of $\tilde{\beta} \lesssim 1$ are consistent with the presence of large grains, and also generally lead to smaller opacities in the millimetric (Miyake \& Nakagawa 1993; D'Alessio et al. 2001). This implies that the larger disk masses derived for GM Aur may be (at least partly) an artefact of the opacity law.

In order to account for the uncertainty on $\kappa_{0}$ we hence consider cases for which the $100 \mathrm{AU}$ gas surface density is either multiplied or divided by a factor 3 compared to the values obtained by Kitamura et al. (2002).

\section{Model description}

Our aim in this paper is to obtain evolutionary models able to satisfy the observational constraints provided by Table 1 and perform a sensitivity analysis of the model parameters for DM Tau and GM Aur. This requires solving the evolution of the gas disk for $\sim 10 \mathrm{Myr}$ and searching not only for the "best" models but for the ensemble of parameters compatible with the observations and their uncertainties. With current (and near-future) computing facilities we must restrict ourselves to a relatively simple system of $1 \mathrm{D}$ radial equations in which all quantities have been vertically averaged and radiative transport is approximated. Consequences of this averaging appear to be relatively mild in terms of accuracy (Huré \& Galliano 2000; see also Ruden \& Lin 1986). This is certainly not a concerning problem, given that we are seeking orderof-magnitude constraints on the turbulent viscosity and other relevant parameters.

We hereafter describe our system of equations in the framework of a simple, but relatively complete model including hopefully - most relevant physical mechanisms. We also discuss its limitations in Sect. 3.5.

\subsection{Viscous evolution of the surface density}

The evolution of a disk follows conservation of angular momentum and mass. As mass is accreted into the inner region of the disk and finally onto the central star, part of the disk material must migrate outwards to conserve angular momentum. If the disk is axisymmetric and geometrically thin, the dynamics are only radially dependent and an effective turbulent viscosity, $v$, can be postulated as the angular momentum transport mechanism. In this case, the equation giving the evolution of the disk surface density, $\Sigma$, can be written as,

$\frac{\partial \Sigma}{\partial t}=\frac{3}{r} \frac{\partial}{\partial r}\left(\sqrt{r} \frac{\partial}{\partial r}[v \Sigma \sqrt{r}]\right)+S(r, t)$

where we have introduced $S(r, t)$, a source term accounting for the accretion from the molecular cloud core onto the circumstellar disk (see Sect. 3.3).

The general solution of (2) is a disk that progressively accretes mass inwards while redistributing a part of the outermost material further away to conserve angular momentum until all of the material is accreted and all of the angular momentum has been transported away (Lynden-Bell \& Pringle 1974). This process is modulated by the value of the (turbulent) viscosity $v$.

\subsection{Two parameterizations of turbulence: $\alpha$ and $\beta$ models}

A prescription for the turbulent viscosity was originally postulated by Shakura \& Sunyaev (1973) and has been extensively used in models of turbulent disks known since then as alpha disk models. According to this prescription,

$v=\alpha c_{\mathrm{s}} H$

The free parameter $\alpha$ controls the amount of turbulence in a turbulent medium where the scale height $H$, and the isothermal sound speed $c_{\mathrm{s}}$, are upper limits to the mixing length and turbulent velocity, respectively. Accretion rates inferred in T Tauri stars are compatible with $\alpha \approx 10^{-2}$ (Hartmann et al. 1998). As a caveat, one should note that it is difficult to imagine any physical process that would yield a viscosity obeying strictly to this parameterization, with $\alpha$ being spatially uniform and temporally constant. In this sense, any value of $\alpha$ that can be retrieved has to be considered as an undefined average over both time and space of the adimensional quantity $v /\left(c_{\mathrm{s}} H\right)$.

A possible source of angular momentum transport that can be approximately parameterized in the $\alpha$-framework is due to the so-called magnetorotational instability (Balbus \& Hawley 1991, 1998, 2000). The instability arises from the fact that ionized elements in a magnetized environment tend to conserve their velocity. In a keplerian magnetized disk, an element being displaced inward will have a lower velocity than other elements at the same location. Therefore, it will also have a smaller angular momentum and it will tend to sink further in (see Terquem 2002, for a nice description of the process). The result is an inner transport of material and an outward transport of angular momentum. Numerical calculations of rotating magnetic disks indicate that a magnetorotational instability can effectively yield a viscosity with $\alpha$ between $10^{-3}$ and 0.1 (Brandenburg et al. 1995; Stone et al. 2000; Papaloizou $\&$ Nelson 2003). The suppression of the instability in neutral regions of the disk could imply rapid spatial variations of $\alpha$ (Gammie 1996).

Another mechanism initially proposed to be responsible for angular momentum transport is thermal convection (Lin \& Papaloizou 1980; Ruden \& Lin 1986). The mechanism had 
largely fallen out of favor after direct hydrodynamical simulations of thermal convection in disks by Stone \& Balbus (1996; see also Stone et al. 2000) had resulted in very small angular momentum transport, mostly inward instead of outward. However, these results are challenged by recent simulations of convection in a baroclinically unstable disk that yield an outward angular momentum transport and corresponding values of $\alpha$ between $10^{-4}$ and $10^{-2}$ (Klahr et al. 1999; Klahr \& Bodenheimer 2001). Turbulent angular momentum transport due solely to thermal convection can be tested by our models because of the particularity that it will cease in an optically thin medium. As proposed by Ruden \& Pollack (1991), turbulent viscosity can then be modeled by a value of $\alpha$ which is uniform in the optically thick part, and goes to zero when the disk becomes optically thin.

Another potential source of angular momentum transport is that due to shear instabilities (e.g. Dubrulle 1993). This mechanism was claimed to be inadequate on the basis of numerical simulations (Balbus et al. 1996; Hawley et al. 1999). However, the disappearance of turbulence in the hydrodynamical simulations may be an artifact due to the limited resolution of the simulations (Longaretti 2002). Indeed, turbulence has been shown to be sustained in Couette-Taylor experiments with outward decreasing angular velocity (Richard \& Zahn 1999). A different prescription for the turbulent velocity may then be applied:

$v=\beta \frac{\partial \Omega}{\partial R} R^{3}$,

where $\Omega$ is the disk rotation rate. Richard \& Zahn found $\beta \sim$ $2 \times 10^{-5}$. Huré et al. (2000) further showed that disk models using this prescription and $\beta \sim 10^{-5}$ are as capable of explaining the observed accretion rates and disks lifetimes as $\alpha$ models. A convenient feature of this turbulent parameterization is that $v$ does not depend on the temperature: the disk's evolution then becomes independent of complex issues related to radiative transfer and opacities.

Other mechanisms can yield angular momentum transport in circumstellar disks without necessarily being amendable to the calculation of a turbulent viscosity. This is for example the case of gravitational instabilities and density waves (e.g. Laughlin \& Różyckza 1996; Laughlin et al. 1998), which may be an important source of disk evolution early on, when the disk/star ratio is still relatively large. It is also the case of bipolar outflows (e.g. Konigl 1989; Casse \& Ferreira 2000). These probably also have an important role during the cloud collapse phase. Although the ejection is limited to $\sim 10 \%$ of the material accreting onto the central star (Calvet 1997), this could nevertheless represent a substantial sink of angular momentum so that Eq. (2) would not be valid in the central region affected by the outflow. Since both of these effects are of importance only during the early evolution phases, neglecting them yields very limited quantitative changes to our results (see Sect. 5.1).

\subsection{Gravitational collapse of an isothermal molecular cloud core and disk growth}

The formation and early evolution of the star+disk system is governed by the collapse of its parent molecular cloud core.
This process remains poorly known (e.g. André et al. 2000), and we choose to model it using several simplifying assumptions. First, we assume that the cloud envelope is isothermal and spherically symmetric. Any given shell of radius $\ell$ and angular velocity $\omega(\ell)$ will collapse onto the disk within the centrifugal radius (the point at which the maximal angular momentum in the shell is equal to the angular momentum in the disk):

$R_{\mathrm{c}}(t)=\frac{\ell(t)^{4} \omega(\ell)^{2}}{G M(t)}$

where $M(t)$ is the mass that has been accreted onto the star+disk system at time $t$. (In the formalism of the self-similar solutions, $t=0$ corresponds to the formation of the central condensation). Formally, Eq. (5) is valid only in the limit when the disk's gravitational potential can be neglected. However, given our lack of knowledge of the collapse phase, this simplification is perfectly justified.

We further assume that angular momentum is conserved during the collapse and that material falling onto the disk finds its way to a location where its angular momentum is equal to that of the (keplerian) disk. With that hypothesis, and given $\dot{M}$, the accretion rate onto the disk, we derive the following source term:

$S(r, t)=\frac{\dot{M}}{\pi R_{\mathrm{c}}^{2}} \frac{1}{8}\left(\frac{r}{R_{\mathrm{c}}}\right)^{-3 / 2}\left[1-\left(\frac{r}{R_{\mathrm{c}}}\right)^{1 / 2}\right]^{-1 / 2}$.

Departures from a central gravitational potential during the collapse phase imply that Eq. (2) does not perfectly conserve the angular momentum of the disk+envelope system. However, once again, the effect is negligeable.

This expression of $S(r, t)$ differs from that obtained from ballistic integrations by Cassen \& Moosman (1981) who showed that the envelope material that encounters the disk has in fact a subkeplerian rotation rate (see also Nakamoto \& Nakagawa 1994). This yields a small additional, outward, angular momentum transport that we choose to ignore in this simulation: as shown in Fig. 1 below, the effect would tend to decrease the surface density in the outer regions by $40 \%$ at most. The turbulent viscosities that are considered have a much more significant effect. Furthermore, this is also relatively small compared to most sources of uncertainties, in particular those related to the cloud collapse itself.

The values of $\dot{M}$ and $R_{\mathrm{c}}(t)$ depend on the mechanisms that led to the collapse of the molecular cloud. A simple and widely used solution is that of Shu (1977). In that case, a self-similar approach to the problem with $\omega(\ell)=\omega_{\text {cd }}$, a constant rotational speed of the molecular cloud, shows that the collapse may proceed from inside-out, with a constant mass accretion:

$\dot{M}=0.975 \frac{c_{\mathrm{s}}^{3}}{G}$,

where $c_{\mathrm{s}}$ is the isothermal sound speed. Because the collapse solution yields $\ell=c_{\mathrm{s}} t / 2$ and using a mean molecular weight $\mu=2.2$, one can show that the centrifugal radius can be written:

$R_{\mathrm{c}}(t) \simeq 53\left(\frac{\omega_{\mathrm{cd}}}{10^{-14} \mathrm{~s}^{-1}}\right)^{2}\left(\frac{T_{\mathrm{cd}}}{10 \mathrm{~K}}\right)^{-4}\left(\frac{M(t)}{1 M_{\odot}}\right)^{3} \mathrm{AU}$ 


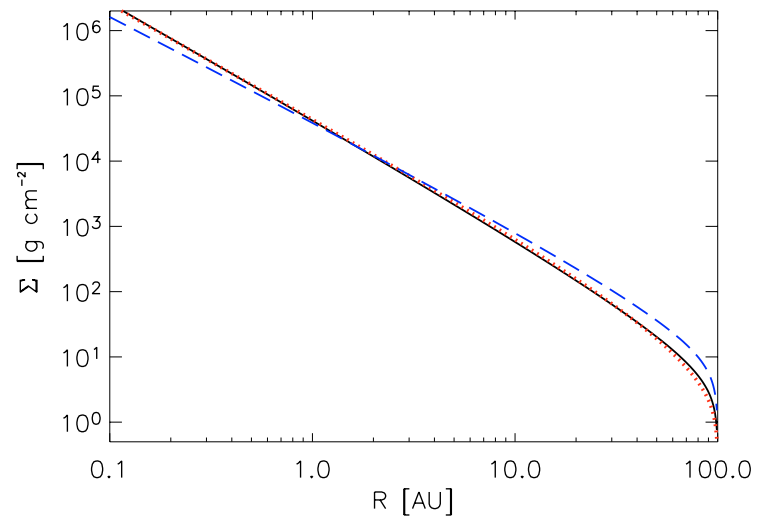

Fig. 1. Surface density obtained at the end of the collapse of a $0.3 M_{0}$ disk, assuming a centrifugal radius $R_{\mathrm{c}}=100 \mathrm{AU}$, and no viscous stress. The plain line corresponds to the collapse of a molecular cloud core in solid rotation. The dotted line shows the same solution when accounting for the outward angular momentum transport due to the subkeplerian momentum of the incoming envelope material (see Cassen \& Moosman 1981). The dashed line corresponds to the collapse of magnetized cloud core with a rotation rate $\omega(\ell) \propto 1 / \ell$.

Observations of hot cores indicate that the accretion rate is not constant over time but enhanced in the very first stages of cloud collapse and progressively diminishing after a more or less long time span of accretion rate close to the one predicted by the Shu theory (Bontemps et al. 1993). However, observations of some very young protostars like IRAM 04191 in the Taurus molecular cloud (Belloche 2002) point to the presence of differential rotation and hence different initial conditions. Indeed, in the presence of a substantial magnetic field, the collapse phase can begin in a cloud whose rotation rate is $\omega(\ell) \propto 1 / \ell$. In that case, accretion proceeds much faster, and a self-similar approach shows that the centrifugal radius then evolves linearly with the accreted mass (Basu 1998):

$\dot{M} \simeq 10 \frac{c_{\mathrm{s}}^{3}}{G}$

$R_{\mathrm{c}}(t) \simeq 15\left(\frac{\omega_{\mathrm{b}}}{10^{-14} \mathrm{~s}^{-1}}\right)^{2}\left(\frac{B_{\mathrm{ref}}}{30 \mu \mathrm{G}}\right)^{-2}\left(\frac{M(t)}{1 M_{\odot}}\right) \mathrm{AU}$,

where $\omega_{\mathrm{b}}$ is the ambient rotation rate of the cloud and $B_{\text {ref }}$ is a background reference magnetic field. The numerical factor in front of the accretion rate is one possible value among many but all models imply it is of order 10 (e.g. Foster \& Chevalier 1993; Basu 1998; Hennebelle et al. 2003). Because the accretion rate also depends on the poorly-known $c_{\mathrm{s}}^{3}$, this uncertainty is ignored.

In the absence of a viscous stress, it can be shown that the collapse of the cloud core yields a profile density $\Sigma(r)=$ $\int S(r, t) \mathrm{d} t$ that is approximately proportional to $r^{-7 / 4}$ in the case of a cloud core in solid rotation and to $r^{-3 / 2}$ in the case of our magnetized, differentially rotating cloud core. The resulting profiles are compared in Fig. 1. Because the centrifugal disk radius at the end of the collapse is fixed in this comparison, the magnetized cloud core has initially more angular momentum: the disk that forms therefore has more mass in its outer regions.

\subsection{Calculating disk properties}

Solving Eq. (2) necessitates the calculation of quantities such as the mid-plane temperature, $T_{\mathrm{c}}$, the vertical scale height, $H$, and the keplerian rotational frequency $\Omega_{\mathrm{k}}$. This has been carried out in many papers (see e.g. Ruden \& Lin 1986; Ruden \& Pollack 1991; Reyes-Ruiz \& Stepinski 1995, to cite only a few), but the detailed expressions may differ slightly from one work to another. Generally, these works have considered disks of small masses and sizes, and have neglected the gravitational potential of the disks themselves. Because this effect may be important for the evolution of DM Tau and GM Aur, we rederive here vertically averaged equations including autogravitation.

\subsubsection{Autogravitation and general disk properties}

We follow Ruden \& Pollack (1991) in expressing the isothermal sound speed, $c_{\mathrm{s}}$, and the mid-plane density, $\rho_{\mathrm{c}}$ as

$c_{\mathrm{s}}^{2}=\frac{\mathcal{R}}{\mu} T_{\mathrm{c}}$,

$\rho_{\mathrm{c}}=\frac{\Sigma}{2 H}$

Here $\mathcal{R}$ is the gas constant, $\mu$ the molecular weight of the gas, supposed to be 2.2 , and $H$ is a measure of the vertical scale height, which is a well defined parameter only in non autogravitating disks. The mass of the disk also affects the keplerian rotational frequencies of the disk:

$\Omega_{\mathrm{k}}(r, t)=\left[\frac{G M_{*}(t)}{r^{3}}+\frac{1}{r} \frac{\mathrm{d} V_{\mathrm{d}}}{\mathrm{d} r}\right]^{1 / 2}$,

where $V_{\mathrm{d}}$ is the gravitational potential of the disk. This potential can be calculated by

$V_{\mathrm{d}}(r)=\int_{R_{*}}^{\infty} \int_{0}^{2 \pi} \frac{-G \Sigma\left(r_{1}\right) r_{1}}{\sqrt{\left(r_{1} \sin \theta\right)^{2}+\left(r-r_{1} \cos \theta\right)^{2}}} \mathrm{~d} r_{1} \mathrm{~d} \theta$.

It is convenient to express Eq. (14) in terms of the radial coordinate alone by using the elliptic integral of the first kind $K[\mathrm{~m}]$ :

$V_{\mathrm{d}}(r)=\int_{R_{*}}^{\infty}-G \frac{4 \mathrm{~K}\left[-\frac{\left.4\left(1+r / r_{1}-1\right)\right)}{\left(r / r_{1}-1\right)^{2}}\right]}{\left|\left(r / r_{1}-1\right)\right|} \Sigma\left(r_{1}\right) \mathrm{d} r_{1}$.

Generally, the incorporation of $V_{\mathrm{d}}$ does not play a major role in the evolution of the disk. Its effect is to slightly increase $\Omega_{\mathrm{k}}$ decreasing the turbulent viscosities through the definitions of $H$ and $v$. Its influence is greater in massive and extended disks with an inner low mass star.

The vertical scale height, $H$, is calculated assuming vertical hydrostatic equilibrium, considering the vertical component of star gravity and the local gravitation of the disk. This is approximated from the infinite and R-homogeneous slab approximation, which considers the locally equivalent limits $\Sigma=$ cte or $z \rightarrow 0$ (Paczyński 1978; Huré 2000):

$\frac{1}{\rho} \frac{\mathrm{d} P}{\mathrm{~d} z}=-\Omega_{\mathrm{k}}^{2} z-4 \pi G \Sigma \equiv g z$

where $g$ is the effective gravity from both star and disk. 
An important simplification is to approximate the disk's vertical structure by an isotherm (the departures from an isotherm yield only second order effect). In that case, the disk pressure and density decrease exponentially:

$(P, \rho)=\left(P_{0}, \rho_{0}\right) \exp \left(-\left(z / H_{0}+\left(z / H_{1}\right)^{2}\right)\right)$,

where the $H_{0}$ term comes from the autogravitation term and $H_{1}$ is the classical scale height coming from the vertical component of the radial gravity of the star. They are defined as

$$
\begin{aligned}
& H_{0}=\frac{c_{\mathrm{s}}^{2}}{4 \pi G \Sigma}, \\
& H_{1}=\frac{\sqrt{2} c_{\mathrm{s}}}{\Omega} .
\end{aligned}
$$

The first one is dominant for large distances and large values of $\Sigma$, where the disk gravitation dominates over the vertical component of the central star gravitation. The second one is the scale height in absence of autogravitation. Consistency with our definition of $\rho_{\mathrm{c}}$ through Eq. (12) requires that $H$ has the following form:

$H=H_{1} \frac{\sqrt{\pi}}{2} \exp \left(\frac{H_{1}}{2 H_{0}}\right)^{2}\left(1-\operatorname{Erf}\left(\frac{H_{1}}{2 H_{0}}\right)\right)$.

When autogravitation is negligible, $H_{0}$ is large and $H \rightarrow$ $\sqrt{\pi} / 2 H_{1}$. When the disk's gravity is significant, $H_{0}$ is small and $H<H_{1}$. The role of the disk gravity is mainly restricted to produce a slight vertical flattening of the outer disk.

The assumption of vertical isothermal structure is generally accurate only close to the disk mid-plane and values of $H$ should be considered only as reasonable estimates instead of exact calculations. This factor yields a slight uncertainty on the magnitude of $v$ in the case of the $\alpha$ parameterization.

\subsubsection{Temperature calculation}

In order to calculate mid-plane temperatures the following assumptions are made:

- the disk is geometrically thin;

- it is heated by the star's illumination but also by the dissipation of viscous energy by turbulence;

- it is assumed to be optically thick in the radial direction everywhere so that heat can be transported efficiently only in the vertical direction.

In thermal equilibrium, the disk's cooling flux is equal to the sum of the heating fluxes due to viscous dissipation and external sources: we define $T_{l}$ as the effective temperature at which the disk is being heated by the central star and external sources and $T_{\mathrm{e}}$ as the emission temperature at which the disk cools down. These quantities are then related by the following expression:

$2 \sigma_{\mathrm{B}} T_{\mathrm{e}}^{4}=\Sigma v r^{2} \Omega_{r}^{2}+2 \sigma_{\mathrm{B}} T_{l}^{4}$,

where $\sigma_{\mathrm{B}}$ is Stephan-Boltzmann's constant, $T_{\mathrm{e}}$ is the effective temperature of the disk, and $T_{l}$ is an effective temperature to which an inert disk would be heated by external sources. $T_{l}$ will be discussed in the next section.
Obtaining the mid-plane temperature $T_{\mathrm{m}}$ from the effective temperature $T_{\mathrm{e}}$ is generally a complex radiative transfer problem. However, in both the optically-thick and opticallythin regimes, analytic expressions can be found (assuming that the opacities behave correctly). In the present work, we adopt the expression derived by Nakamoto \& Nakagawa (1994), who approximate the mid-plane temperature as a sum of opticallythick and optically-thin contributions:

$\sigma_{\mathrm{B}} T_{\mathrm{c}}^{4}=\frac{1}{2}\left(\frac{3}{8} \tau_{\mathrm{R}}+\frac{1}{2 \tau_{\mathrm{p}}}\right) \Sigma v r^{2} \Omega_{r}^{2}+\sigma_{\mathrm{B}} T_{l}^{4}$,

where $\tau_{\mathrm{R}}$ and $\tau_{\mathrm{P}}$ are the Rosseland and Planck mean optical depths respectively. The optical depth from the midplane to the surface of the nebula, $\tau_{\mathrm{R}}$, is defined in terms of the Rosseland mean opacity $\kappa_{\mathrm{R}}\left(\rho_{\mathrm{c}}, T_{\mathrm{c}}\right)$ by

$\tau_{\mathrm{R}}=\frac{\kappa_{\mathrm{R}} \Sigma}{2}$.

To evaluate $\kappa_{R}$, we used the Rosseland mean opacity of dust grains provided by Pollack et al. (1986) in the form of a power law: $\kappa_{\mathrm{R}}\left(\rho_{\mathrm{c}}, T_{\mathrm{c}}\right)=K_{0} \rho_{\mathrm{c}}{ }^{m} T_{\mathrm{c}}{ }^{n}$. Note that the rapid changes of the opacities in different regimes (e.g. due to the evaporation of water,... etc.) imply that a robust autoconsistent numerical method must be used to solve the temperature equation. More recent opacities provided by Hartmann \& Kenyon (1996) and Bell \& Lin (1994) seem to be in general agreement with the values of Pollack et al. As Nakamoto \& Nakagawa (1994), we further assumed $\tau_{\mathrm{P}}=2.4 \tau_{\mathrm{R}}$.

Since the intensity of the turbulence viscosity is determined by the disk temperature and determines itself part of the heating the set of nonlinear equations must be solved simultaneously in an autoconsistent manner once a value of $\alpha$ is given. A timeforward integration of the density surface distribution is then possible. The problem is more straightforward in the case of the $\beta$ prescription because $v$ is then independent of temperature.

\subsubsection{Irradiation from the central star}

Stellar photons reprocessed by the accretion disk constitute a significant contribution to the disk's thermal budget, especially in regions where dissipation by viscous processes is small, i.e. mostly in the external regions of the disk. Again, a proper treatment of stellar irradiation is beyond the scope of this work, but we attempt to capture the essential underlying physics under our 1D approach.

At distances much larger than the stellar radius, it can be shown that stellar irradiation implies an effective temperature that is a function of the star's effective temperature $T_{*}$ and radius $R_{*}$ (Adams et al. 1988; Ruden \& Pollack 1991):

$T_{l_{1}}=T_{*}\left[\frac{2}{3 \pi}\left(\frac{R_{*}}{r}\right)^{3}+\frac{1}{2}\left(\frac{R_{*}}{r}\right)^{2}\left(\frac{H}{r}\right)\left(\frac{\mathrm{d} \ln H}{\mathrm{~d} \ln r}-1\right)\right]^{1 / 4}$.

The first term inside the brackets is the contribution due to the finite-sized star irradiating a flat disk. The second term, proportional to ( $\mathrm{d} \ln H / \mathrm{d} \ln r-1)$ accounts for the flaring of the disk. We find numerically that this factor becomes significant only 


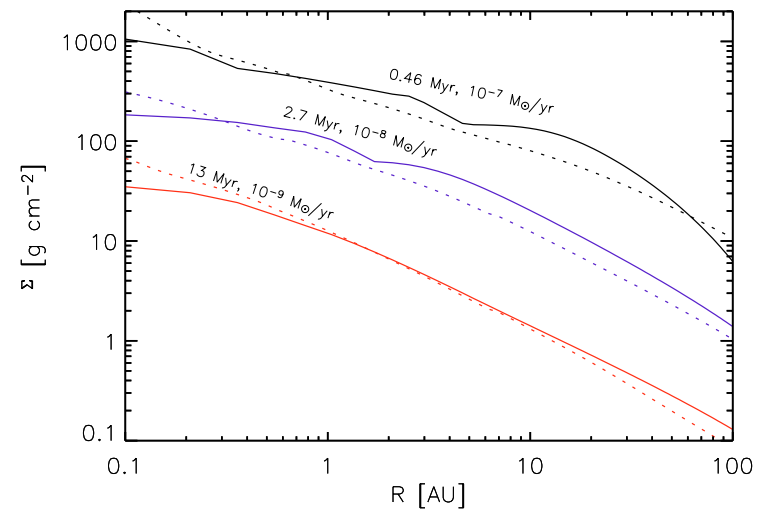

Fig. 2. Surface density as a function of orbital distance for three different times, corresponding to accretion rates onto the central protostar of $10^{-7}, 10^{-8}$ and $10^{-9} M_{\odot} \mathrm{yr}^{-1}$. Plain lines show the results of our model. These are compared to similar calculations by D'Alessio et al. (2001) for a standard $\alpha$-disk with $\alpha=0.01, M_{*}=0.5 M_{\odot}$, $R_{*}=0.0093 \mathrm{AU}$ and $T_{*}=4000 \mathrm{~K}$ (dotted lines). We further used: $M_{0}=0.2 M_{\odot}, \omega_{\mathrm{cd}}=3 \times 10^{-3} \mathrm{~s}^{-1}$ and $T_{\mathrm{cd}}=10 \mathrm{~K}$ implying a total accretion time of the cloud core of $0.3 \mathrm{Myr}$ and an outer maximum centrifugal radius of $60 \mathrm{AU}$.

for radii $r>50 \mathrm{AU}$. However, this factor is a significant source of numerical instabilities. This is an interesting problem that certainly requires further work. At present, we choose to avoid it by imposing

$\mathrm{d} \ln H / \mathrm{d} \ln r=9 / 7$,

which corresponds to the (approximate) equilibrium solution for a disk whose temperature is dominated by the flaring term (Chiang \& Goldreich 1997). We verified that this hypothesis is autoconsistent, i.e. our disks are close to $H \propto r^{9 / 7}$ in regions where the flaring term dominates.

We additionally considered that the disk is heated by its environment which radiates at the same temperature as the molecular cloud, $T_{l_{2}}=T_{\text {cd }}$. The true irradiation temperature $T_{l}$ is then computed as

$T_{l}^{4}=T_{l_{1}}^{4}+T_{l_{2}}^{4}$.

\subsection{Comparison to an $\alpha$-disk model with detailed radiative transfer}

Our model was tested against a calculation by d'Alessio et al. $(1999,2001)$ for a $0.5 M_{\odot}$ T-Tauri star. Their calculation of the density profile is based on a standard $\alpha$-disk model, in which a static solution to Eq. (2) with no source term is found by imposing a constant mass flux throughout the disk. As shown in Fig. 2, the resulting surface densities for a given accretion rate onto the star differ slightly. This is not unexpected, due to the fact that we included the collapse phase, because assumed opacities are different, and because the static solution is not necessarily a good estimate of the disk's structure, especially at large orbital distances.

Despite these differences, the midplane temperatures inferred in our model compare well to the more elaborate $2 \mathrm{D}$ calculations by D'Alessio et al. Figure 3 shows that the

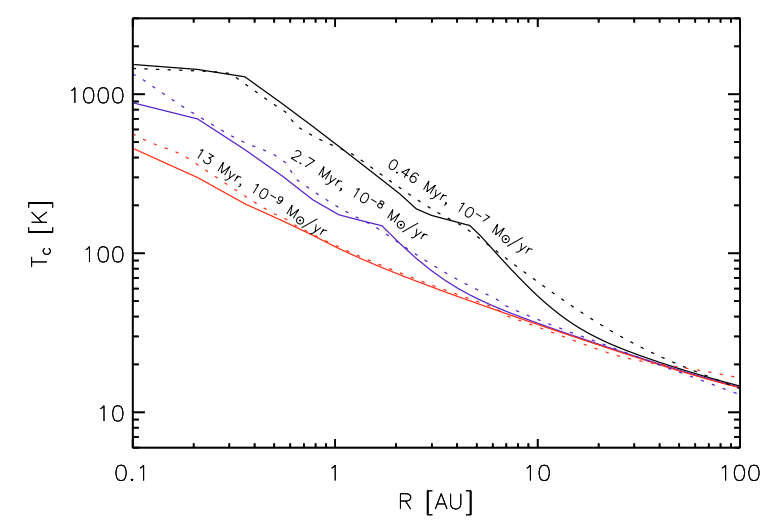

Fig. 3. Midplane temperatures as a function of orbital distance calculated in this work (plain lines) and by D'Alessio et al. (2001) (dotted lines), in the same conditions as in Fig. 2.

comparison is good in two regimes: (i) In the inner parts of the disk $(R \lesssim 10 \mathrm{AU})$, where the release of gravitational energy by viscous dissipation dominates over the star's irradiation and governs the disk's temperature structure; (ii) In the intermediate and outer parts of the disk, where irradiation from the central star is the dominant source of heating and the temperature profile tends towards $T(r) \propto r^{-1 / 2}$.

\subsection{Limitations of the model}

\subsubsection{Structure of the inner disk}

In the calculations that are presented, we assume that the disk extends from an arbitrary inner boundary at $0.1 \mathrm{AU}$ and beyond. The exact location of this inner boundary is of little importance for the evolution of the outer disk (say, beyond $10 \mathrm{AU}$ ). However, the exact structure of the inner disk and the fact that recent observations seem to point to a clearing of the region inside $\sim 5 \mathrm{AU}$ (Bergin et al. 2004) can affect the constraints derived from the accretion rate. For example, it can be noticed that the presence of a Jupiter-mass planet would lower the accretion rate onto the star, because part of the material from the outer disk would be accreted by the planet (e.g. D'Angelo et al. 2002; Bate et al. 2003), and part of the disk's gravitational energy would be used to push the planet inward. Clearly, in the absence of detailed models of this and because of other possible explanations (photoevaporation, grain growth) it would be unrealistic to try to also fit the observed structure of the inner disk with our simple model. Last but not least, the error bar on $\dot{M}$ is relatively large and is presumably larger than this source of uncertainty.

\subsubsection{Thermal vertical structure}

In models with an $\alpha$-viscosity, the temperature determines the sound speed $c_{\mathrm{s}}$, the scale height $H$ and hence the viscosity $v$ itself. We use the midplane temperature because the gravity near the midplane is low $(\sim \omega z)$ and vertical temperature variations should be small in these regions. However, radiative transfer models (Malbet \& Bertout 1991; Chiang \& Goldreich 1997; 
D'Alessio et al. 1999, 2001) show that in regions where the stellar irradiation is significant, the temperature in the outer layers of the disk is expected to be larger than at the midplane. This was verified by (Dartois et al. 2003) who found that in DM Tau's outer disk, the central temperature is $T_{\mathrm{c}} \sim 15 \mathrm{~K}$ while $T_{\text {photosphere }} \sim 30 \mathrm{~K}$.

Another aspect of the problem is that in the tenuous atmosphere of the outer disk, the gas may not be in thermal equilibrium and may therefore be heated more than the dust disk, i.e. by absorption of FUV photons. Altogether, this should introduce a maximum of a factor of 2 indetermination on the temperatures and $\alpha$-viscosities of the outer optically thin disk.

\subsubsection{Non-viscous processes affecting the disk evolution}

Our model assumes that the present disks around DM Tau and GM Aur are solely the result of the collapse of a molecular cloud core and of the subsequent viscous evolution of the disk. May these disks be altered by other physical processes? Following Hollenbach et al. (2000), we identify five possible sources of disk dispersal:

- wind stripping;

- photoevaporation due to an external source;

- photoevaporation due to the central star;

- tidal stripping due to close stellar encounters;

- planet formation.

Wind stripping may indeed affect the disk thickness, but according to Hollenbach et al. (2000), the associated timescales for disk dispersal are, in the regions of interest, well over $10 \mathrm{Myr}$ and can be neglected.

Unlike in Orion (e.g. O'Dell et al. 1993), photoevaporation due to external sources should not be a concern in the TaurusAuriga association due to the small number of massive stars present.

Photoevaporation due to the central star may be estimated from the work of Hollenbach et al. (1994; see also Shu et al. 1993). The authors estimate that the total mass loss due to UV photoevaporation of disk material is:

$\dot{M}_{\mathrm{UV}}=4.1 \times 10^{-10} \Phi_{41}^{1 / 2} M_{*}^{1 / 2} M_{\odot} \mathrm{yr}^{-1}$,

where $\Phi_{41}$ is the ionizing photon luminosity of the central star in units of $10^{41} \mathrm{~s}^{-1}$. There is no precise value of this parameter, but Alexander et al. (2005) advocate using the observed $\mathrm{He}$ II/C IV line ratio to derive the hardness of the ultraviolet spectrum and obtain $\Phi_{41} \approx 1-1000$ for five classical T-Tauri stars. As shown by Clarke et al. (2001) and Matsuyama et al. (2003), photoevaporation alters significantly the inner disk's structure when $\dot{M}_{\mathrm{UV}} \sim \dot{M}_{*}$, where $\dot{M}_{*}$ is the accretion rate onto the star. This implies that photoevaporation may play a role even in DM Tau and GM Aur if the UV photon rate is in the upper range of that estimated by Alexander et al. Even in that case however, we expect the effect on the structure of the outer disks to be relatively modest.

Tidal stripping may be important for the evolution of accretion disks in relatively dense environments. A simple estimate for the timescale of truncation of a disk to a radius $r_{\mathrm{d}}$ assumes that the disk is stripped to about $1 / 3$ the impact parameter (Clarke \& Pringle 1991; Hollenbach et al. 2000); hence: $t_{\mathrm{SE}} \approx 1 /\left(n_{\star} \sigma v\right)$, where $n_{\star}$ is the density of stars, $\sigma \approx \pi\left(3 r_{\mathrm{d}}\right)^{2}$ is the collision cross section and $v$ is the velocity dispersion of stars. In our case, assuming $t_{\mathrm{SE}} \lesssim 10^{7} \mathrm{yr}, v \approx 1 \mathrm{~km} \mathrm{~s}^{-1}$ and $r_{\mathrm{d}} \approx 1000 \mathrm{AU}$ implies that stellar encounters could become important for star densities $n_{\star} \gtrsim 50 \mathrm{pc}^{-3}$. Values $n_{\star} \approx 100 \mathrm{pc}^{-3}$ appear to be typical of the central Taurus cloud (Clarke et al. 2000). Moreover, both DM Tau and GM Aur are relatively isolated in this cloud and consequently this mechanism has probably not affected their disks. This is probably unlike other young stars, which may explain why young stars with very extended disks remain relatively rare.

Last but not least, planet formation is a mechanism susceptible of greatly modifying the structure of disks, particularly when giant planets form. However, planets are thought to form closer to the central star than can be probed by the present observational techniques. Their effect on the disks can be thought as a surface density sink, and should hence be relatively modest at large orbital distances.

\subsubsection{Gravitational stability of the disk}

The local gravitational stability of a rotating disk against axisymmetric perturbations is measured by the Toomre- $Q$ parameter, which is defined by

$Q=\frac{k c_{\mathrm{s}}}{\pi G \Sigma}$

where $k$ is the epicyclic frequency given by $k^{2}=$ $\left(1 / r^{3}\right)\left[\partial\left(r^{4} \Omega_{\mathrm{k}}^{2}\right) / \partial r\right]$ (Toomre 1964; Goldreich \& Lynden-Bell 1965). Disks are gravitationally stable if $Q>1$ all over the disk (the rotation gradient dominates and the disk follows axisymmetric evolution) and are unstable if $Q \leq 1$ anywhere (gravitation may dominate locally and break out the axisymmetry). In this case disks may produce a direct gravitational collapse of particles into planetesimals (Goldreich \& Ward 1973), giant planet cores (Boss 2003) or may develop spiral density waves able to redistribute the angular momentum in a non local way until stability is reached again (Laughlin \& Różyczka 1996; Laughlin et al. 1997, 1998). This last case is the generally accepted scenario for the evolution of massive disks.

Nakamoto \& Nakagawa (1994) showed that disk instabilities are more likely to appear in disks with low values of $\alpha$ and high values of $\omega_{\text {cd }}$. Laughlin \& Różyczka (1996) found that in most of the situations the global transport of angular momentum by spiral arms in gravitationally unstable disks may be roughly in agreement with axisymmetric models with values of $\alpha_{\mathrm{s}}$ of $0.02-0.03$.

Although both DM Tau and GM Aur disks are at present gravitationally stable ( $Q>1$ everywhere), they may have gone through an early unstable phase. In order to account for this effect, we adopted $\alpha=\operatorname{Max}(0.03, \alpha)$ when the condition $Q<1$ was met somewhere in the disk. A limited number of models with $\alpha \geq 0.03$ went through an unstable phase without any artificial increase in their viscosity. We did not modify the viscosity of $\beta$-models, whatever the value of $Q$. 
We observed a posteriori that the gravitational instability phase was generally limited in time to less than $0.1 \mathrm{Myr}$. An incorrect handling of gravitational instabilities hence yields a negligible error on the age of the system. An effect that is potentially more significant, and ignored in the present study, concerns the fact that the advection of disk material can depart from a diffusive solution. To the extreme, this process may lead to the formation of stellar or planetary companions. Clearly, a more consistent treatment of gravitational instabilities would be desirable.

\section{Fitting the observations}

\subsection{Setting of the calculations}

The equations described in the previous section are solved numerically using an explicit finite-difference scheme. Each calculation begins at time $t=M_{0} / \dot{M}$. The inner disk boundary is set to $R_{\text {in }}=0.1 \mathrm{AU}$, and the maximum computational space ends at $R_{\text {out }}=10^{4} \mathrm{AU}$. In order to solve numerically Eq. (2) we follow the method described by Bath \& Pringle (1981). This requires the radial points to be equally spaced on a $X=\sqrt{r}$ space. The stability condition required to solve Eq. (2) is that the time-step $\Delta t$ obeys

$\Delta t \geq \operatorname{Min}\left(\frac{X^{2} \Delta X^{2}}{24 v}\right)$.

An adaptive time-step scheme taking into account this condition is used. A grid resolution of 250 points is adequate for disks with a centrifugal radius that is always larger than $4 \mathrm{AU}$. To calculate the evolution of disks with smaller values of $j_{\mathrm{cd}}$ so that $2 \lessgtr R_{\mathrm{c}} \lessgtr 4 \mathrm{AU}$, we use 500 points, corresponding to a resolution in the inner regions $\sim 0.15 \mathrm{AU}$.

The model parameters are:

- $\alpha$ or $\beta$, which characterize the magnitude and functional form of the turbulent viscosity;

- $\omega_{\mathrm{cd}}$, the angular velocity of the molecular cloud core;

- $T_{\text {cd }}$, the temperature of the molecular cloud (which is equivalent to a characteristic accretion velocity from the molecular cloud onto the circumstellar disk);

- $M_{0}$, the initial mass of the protostar;

- $M_{\mathrm{cd}}$, the total amount of material initially in the molecular cloud and eventually in the star+disk system.

These parameters reflect either uncertainties in the physics of star formation $\left(\alpha-\beta, M_{0}\right)$ or unknown initial conditions $\left(\omega_{\mathrm{cd}}, M_{\mathrm{cd}}\right)$, or both $\left(T_{\mathrm{cd}}\right.$ through the accretion rate of the molecular cloud, see Eq. (7)).

Our approach is to constrain these parameters by extensive numerical simulations and comparison with observations. Four sets of calculations are performed for DM Tau and GM Aur, using either the $\alpha$ or $\beta$ parameterizations and considering the Shu (1977) classical cloud collapse model. To explore the sensitivity of the results to the cloud collapse model, we calculate a fifth set of model for the DM Tau $\alpha$ case and the collapse scenario derived from Basu (1998).

In order to efficiently explore the space of parameters we use a Monte-Carlo procedure to chose the values of the model
Table 2. Explored parameter space.

\begin{tabular}{lll}
\hline \hline & DM Tau & GM Aur \\
\hline$M_{0}\left[M_{\odot}\right]$ & 0.05 and 0.30 & 0.05 and 0.40 \\
$M_{\mathrm{cd}}\left[M_{\odot}\right]$ & $0.4-1.0$ & $0.4-1.5$ \\
$\omega_{\mathrm{cd}}\left[\mathrm{s}^{-1}\right]$ & $10^{-15}-10^{-11}$ & $10^{-15}-10^{-11}$ \\
$T_{\mathrm{cd}}[\mathrm{K}]$ & $3.0-30.0$ & $3.0-30.0$ \\
Accretion Time $\left.{ }^{*}\right)[\mathrm{yr}]$ & $45000-5 \times 10^{6}$ & $49000-5 \times 10^{6}$ \\
$\alpha$ & $10^{-5}-1$ & $10^{-5}-1$ \\
$\beta$ & $10^{-6}-0.1$ & $10^{-6}-0.1$ \\
Number of models & $2000 \alpha$ & $2000 \alpha$ \\
& $1500 \beta$ & $1500 \beta$ \\
Magnetic cloud core & $2000 \alpha$ & \\
\hline
\end{tabular}

${ }^{*}$ ) Here the accretion time refers to the time it takes for the molecular cloud core to entirely collapse onto the disk (see Eq. (7)).

parameters within the range given in Table 2. Two values of $M_{0}$ are chosen. A small value is representative of cases in which the central condensation grows relatively slowly, and gravitationnal instabilities dominate the early disk evolution. A large value is representative of an initially evolved situation in which the proto-star forms rapidly, and the disk grows significantly only at later times. This could occur if ambipolar diffusion and jets allow an efficient outward transport of angular momentum early on during the collapse of the molecular cloud.

Cases for which the initial centrifugal radius $R_{\mathrm{c}}$ (see Eq. (5)) is larger than $\sim 5000 \mathrm{AU}$ are disregarded, as well as those with final centrifugal radius too small to solve accurately the building-up of the disk. For numerical reasons models that require a time-integration with a step smaller than $0.5 \mathrm{yr}$ are not calculated. Table 2 provides a summary of the different sets of calculations run and the coverage of the space of parameters. Table 3 provides the numerical limitations imposed by the two different grid resolutions, the minimum time-step required, and the resulting limits on $j_{\mathrm{cd}}$ and $v_{0}$. The values of $j_{\mathrm{cd}}$ bracket inferred values for Class I protostellar envelopes, $10^{20} \lesssim j_{\mathrm{cd}} \lesssim 10^{21} \mathrm{~cm}^{2} \mathrm{~s}^{-1}$ (Ohashi et al. 1997). The upper limit on $v_{0}$ is relatively large and limits us only for extreme values of the $\beta$-parameter in the case of DM Tau (see Fig. 14 hereafter).

The calculations were performed on an 8PCs Linux/Beowulf cluster over several months. The low $\Delta t$ required by the stability criterion imply total computation times for each model that range between minutes to several hours for the most computationally expensive models (specifically, these correspond to 500-points, $\beta$ models of GM Aur). The large database of resulting models is then compared to the various observational constraints in order to extract the possible values of the main physical parameters.

\subsection{Selecting models from observational constraints}

The observational constraints characterizing DM Tau and GM Aur have very different origins. Three independent constraints are used (see Sect. 2):

(a) The fact that the disks are optically thick in ${ }^{12} \mathrm{CO}$ at $100 \mathrm{AU}$ and the outer disk radius requires that $\Sigma$ be larger than a 
Table 3. Numerical limitations of the calculation

\begin{tabular}{ll}
\hline \hline$N_{\text {points }}=250$ & $N_{\text {points }}=500$ \\
\hline$\Delta R_{0}=0.4$ & $\Delta R_{0}=0.15$ \\
$2.3<R_{\mathrm{c}}<5000$ & $0.8<R_{\mathrm{c}}<5000$ \\
$19.0<\log _{10}\left(j_{\mathrm{cd}}\right)<21$ & $18.8<\log _{10}\left(j_{\mathrm{cd}}\right)<21$ \\
\hline
\end{tabular}

$$
\Delta t>0.5 \Rightarrow
$$

$\log _{10}\left(v_{0}\right)<17.2$

$\log _{10}\left(v_{0}\right)<16.5$

$\Delta R_{0}$ is the spatial resolution of the model at the inner disk boundary and $R_{\mathrm{c}}$ is the centrifugal radius, both are given in $\mathrm{AU}, j_{\mathrm{cd}}$ is the specific angular momentum of the molecular cloud and is given in $\mathrm{cm}^{2} \mathrm{~s}^{-1}$, $\Delta t$ is the minimum allowed computational time-step in yr and is related with $v_{0}$ the value of the disk viscosity at the inner boundary given in $\mathrm{cm}^{-2} \mathrm{~s}^{-1}$. The inner disk boundary is located at $0.1 \mathrm{AU}$.

minimal value. This value is calculated by assuming that all $\mathrm{C}$ is in form of vapor $\mathrm{CO}$ and translates into a minimal value of $\Sigma$ at $R_{\text {out }}$.

(b) Values of $\Sigma$ at $100 \mathrm{AU}$ have to be consistent with those inferred from dust emission, allowing for uncertainties in the opacity coefficient, grain size and dust to gas ratio.

(c) The accretion rate onto the star has to fit constraints obtained from the star's excess luminosity. A conservative error bar of 0.54 in $\log _{10}(\dot{M})$ is assumed.

In order to further narrow the possible solution ensemble, we choose to add the following reasonable constraints:

(d) At the outer ${ }^{12} \mathrm{CO}$ radius, the value of $\Sigma$ cannot be larger than 20 times the lower limit. This value is inspired by the discrepancy between the $\mathrm{CO}$ and dust emission observations at $100 \mathrm{AU}$.

(e) The inferred accretion rate is supposed to be known within a realistic but model dependent error bar of only 0.3 in $\log _{10}(\dot{M})$.

These constraints are schematically shown in Fig. 4. They should be further refined by combining observational and theoretical studies. In this work, we purposely use rather unrestrictive interpretations of the observations to obtain robust constraints on the desired physical parameters.

Together with the constraints on the star's mass and age, we thus derive five sets of solutions, from weaker to stronger constraints:

$\{1\}:$ (a)

$\{2\}:(a)+(b)$

$\{3\}:(a)+(b)+(c)$

$\{4\}:(a)+(b)+(c)+(d)$

$\{5\}:(a)+(b)+(c)+(d)+(e)$.

We will mostly discuss the results obtained with sets $\{4\}$ and $\{5\}$.

\subsection{Examples of models behavior: disk history and surface density-temperature evolution}

Before analyzing our global results, it is useful to describe the general behavior of the models in terms of two specific

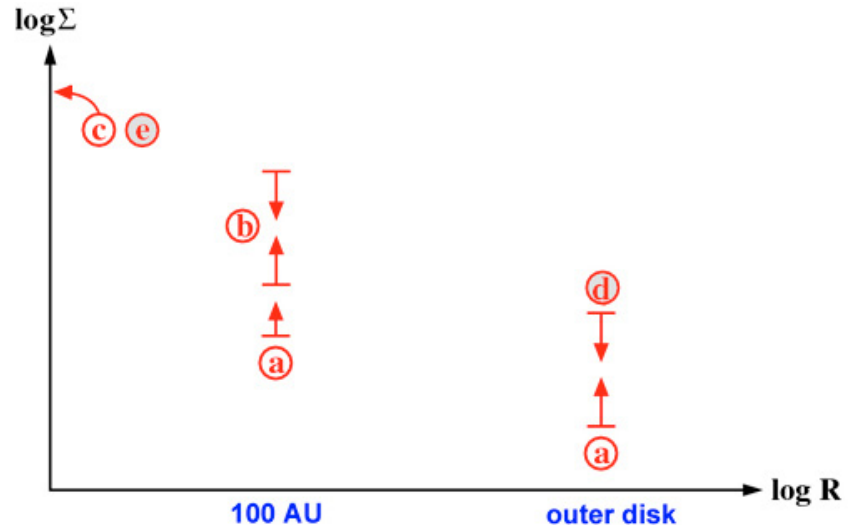

Fig. 4. Illustration showing the different observational constraints used in this work, in terms of surface density and orbital distance. The labels "c" and "e" correspond to constraints on the accretion rate onto the central star. Constraints "e" and "d" are reasonable expectations of the mass accretion rate and outer disk maximum surface density.

examples. We explain here how the observational constraints are used to select a model as a successful fit to the data. We consider two examples based on an $\alpha$-model of DM Tau. The parameters for both examples are listed in Table 4

In all cases, our story starts with a protostar of mass $M_{0}$, no disk and a reservoir of cloud material with mass $M_{\mathrm{cd}}-M_{0}$. Material falls from the molecular cloud at a constant rate inside a disk of growing size $R_{\mathrm{c}}$, a consequence of angular momentum conservation and the inside-out collapse of a molecular cloud core in solid rotation. Because viscous diffusion is more efficient than the increase of $R_{\mathrm{c}}$ with time, the disk spreads beyond the centrifugal radius. The disk can be quite hot in this early phase, especially when $R_{\mathrm{c}}$ is small and the accretion rate is large (large $T_{\mathrm{cd}}$ ). The disk grows in mass until all of the available mass in the molecular cloud core has been accreted to the disk. After that, the central star continues to accrete disk material while the disk expands and cools.

Starting with Example 1, Fig. 5 illustrates the evolution of the star mass, disk mass, centrifugal radius and star accretion rate. Figure 6 shows the evolution of the surface density of the disk. The disk construction phase (dotted lines) appears as a quick phase in which the disk density increases by orders of magnitude with a relatively sharp outer edge. When the molecular cloud has collapsed entirely (at $t=0.18 \mathrm{Myr}$ ), the disk has already spread further than the maximum centrifugal radius (11 AU), a consequence of the relatively large $v$. The later evolution of the system is characterized by the inner accretion of disk material and the outer diffusion of angular momentum. After $3 \mathrm{Myr}$, the diffusion of material proceeds much more slowly due to the lower density, temperature and viscosity of the inner disk.

The midplane temperatures are shown in Fig. 7 for the same time-sequence. The disk heats up considerably during the molecular cloud collapse phase (the first $0.2 \mathrm{Myr}$ ) and cools down gradually when the collapse ceases. The sharp transitions in the temperature curves arise from the different regimes of Rosseland opacities at different temperatures (Ruden \& Pollack 1991). The outer disk beyond $50 \mathrm{AU}$ is optically thin, vertically 
Table 4. Model parameters for examples 1 and 2.

\begin{tabular}{llll}
\hline \hline Parameters & Example 1 & Example 2 & Units \\
(fixed) & & & \\
\hline$\alpha$ & 0.01 & 0.025 & - \\
$\omega_{\text {cd }}$ & $2.3 \times 10^{-14}$ & $2.6 \times 10^{-13}$ & $\left(\mathrm{~s}^{-1}\right)$ \\
$T_{\mathrm{cd}}$ & 14 & 17 & $(\mathrm{~K})$ \\
$M_{0}$ & 0.05 & 0.05 & $\left(M_{\odot}\right)$ \\
$M_{\text {cd }}$ & 0.515 & 0.585 & $\left(M_{\odot}\right)$ \\
\hline (derived) & & & \\
\hline $\log _{10}\left(J_{\mathrm{cd}}\right)$ & 52.3 & 53.4 & $\left(\mathrm{~g} \mathrm{~cm}^{2} \mathrm{~s}^{-1}\right)$ \\
$\log _{10}\left(j_{\mathrm{cd}}\right)$ & 19.3 & 20.3 & $\left(\mathrm{~cm}^{2} \mathrm{~s}^{-1}\right)$ \\
$R_{\mathrm{c}}$ & 11 & 830 & $(\mathrm{AU})$ \\
Accretion time & 0.18 & 0.15 & $(\mathrm{Myr})$ \\
\hline
\end{tabular}

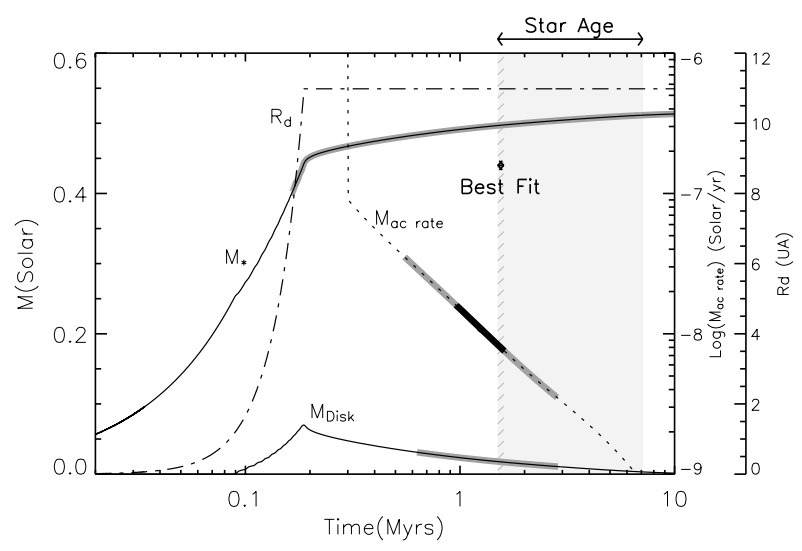

Fig. 5. Example 1. Evolution of star mass $M_{*}$ and disk mass $M_{\text {disk }}$ as a function of time with masses in solar units (corresponding axis to the left) for the model parameters of Table 4 . The accretion rate onto the central star is shown as a dotted line (corresponding axis: first to the right). The evolution of the centrifugal radius $R_{\mathrm{c}}$ (see Eq. (5)) is shown as a dash-dotted line (corresponding axis: far-right). Gray curves and the hashed region indicate time sequences when selected observational constraints are verified (see text).

extended, flared, and its thermal structure is determined solely by stellar irradiation and a diffuse heat source of $T_{\mathrm{cd}}$.

In Example 1, the disk is always stable to gravitational perturbations $(Q>1)$ and the disk mass is never a large fraction of the star mass. This model evolves smoothly with values of the initial parameters in good agreement with expected values in the Taurus Aurigae region. The question then is: does this model satisfy the observational requirements for DM Tau? And in that case, what other values of the set of parameters representative perhaps of very different initial conditions or turbulence in the disk, would also agree with the observations?

Figure 5 shows thick grey lines superimposed on each plotted quantity. Each one shows the range of time for which that quantity agrees with the available observations. For $M_{\text {Disk }}$, the grey line represents the period of time when the $\Sigma$ surface density satisfies the observational error bars discussed in Sect. 2. This period is easily identified in Fig. 6. The accretion rate is reproduced either within large error bars (thick grey line), or with

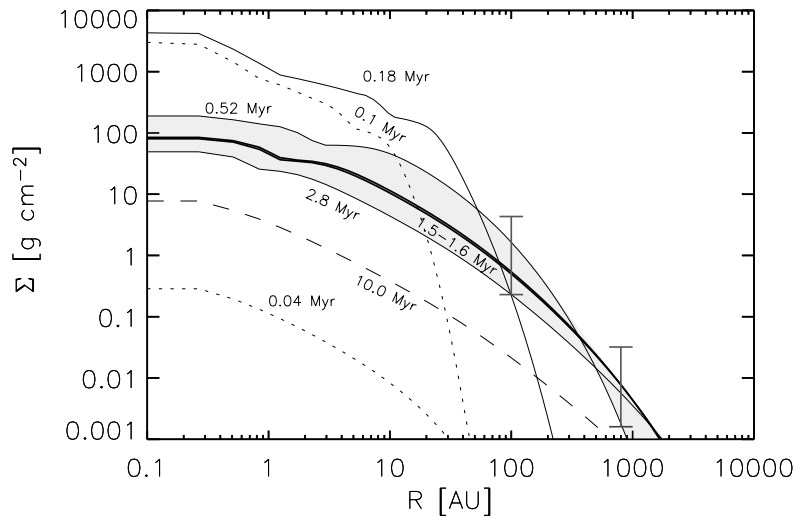

Fig. 6. Example 1. Surface density versus orbital distance at different times for the model parameters of Table 4. Dotted lines correspond to the early formation of the disk. The collapse of the molecular cloud ends after 0.18 Myr. The dashed line at $10 \mathrm{Myr}$ corresponds to the end of the simulation. The error bars represent the $\Sigma$ values at $100 \mathrm{AU}$ and in the outer radius that are used as observational constraints for DM Tau. The gray area shows the ensemble of models fitting those constraints. The dark-shaded region shows the $\Sigma$ distribution at the time-lapse when all observational constraints are satisfied (see text).

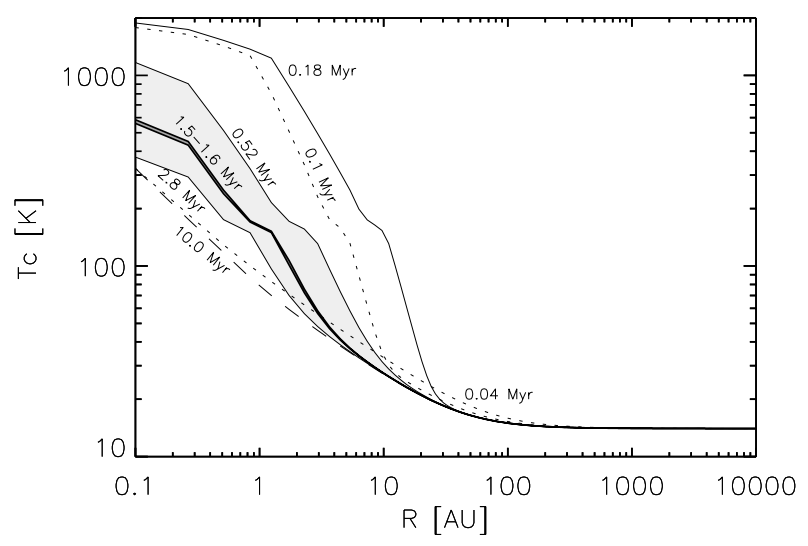

Fig. 7. Example 1. Same as Fig. 6, but for the midplane temperature as a function of orbital distance.

small error bars (thick black line) (see Sect. 2). The uncertainty over the star age for DM Tau is marked as a light-grey box. Figure 5 hence shows that the model is a good fit to the data from 1.5 to $2.8 \mathrm{Myr}$ (surface densities and star age), from 1.5 to $2.6 \mathrm{Myr}$ ( $\dot{M}$ with its large error) or from 1.5 to $1.6 \mathrm{Myr}$ (small error bars on $\dot{M}$ ). The latter is shown as a dashed region.

This model hence does fulfill the "strict" observational constraints (set $\{3\}$ ) and also the reasonable additions (sets $\{4\}$ and $\{5\})$. This example shows how DM Tau's 800 AU disk can be formed by viscous diffusion of an initially much smaller disk, with a centrifugal radius $R_{\mathrm{c}}=11 \mathrm{AU}$.

Let us now examine our second example. Here the disk is assumed to form in $0.15 \mathrm{Myr}$ with a maximum centrifugal radius of $800 \mathrm{AU}$. Most of the disk material falls so far from the central star that the disk gets more massive than the central star at the end of the collapse (Figs. 8 and 9). Yet, the disk diffuses outwards and gets accreted into the central star. For a certain period of time (4-5.4 Myr) it also satisfies all the observational constraints we have considered. 


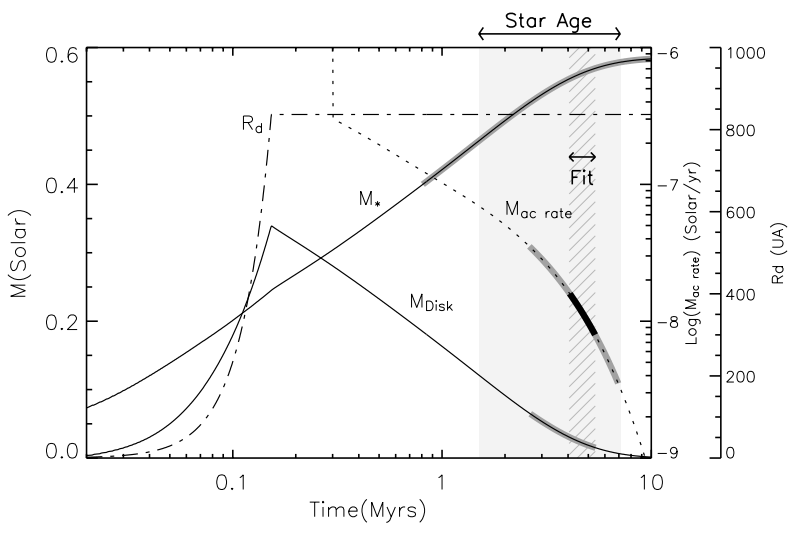

Fig. 8. Example 2. Evolution of star mass $M_{*}$ and disk mass $M_{\text {disk }}$ as a function of time. See Fig. 5 and Table 4 for details.

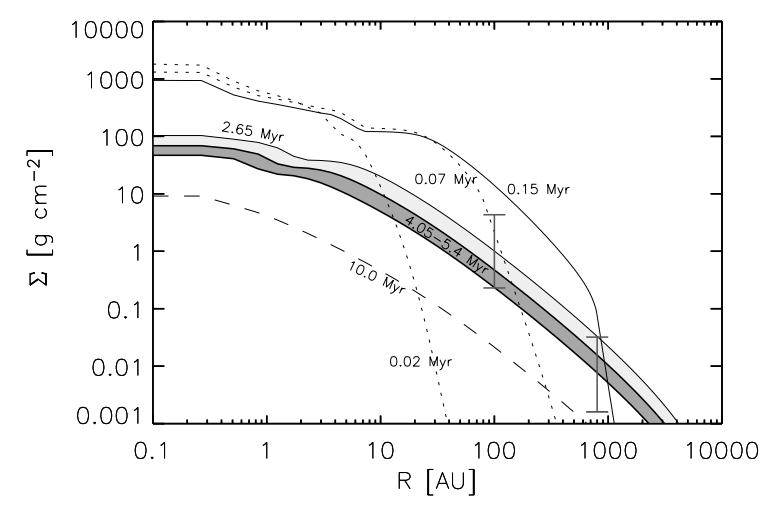

Fig. 9. Example 2. Surface density versus orbital distance at different times. See Fig. 6 and Table 4 for details.

Observationally it may be very difficult to distinguish between these two scenarios at a late and evolved age but each one has very different implications for the formation of planetary systems. In the first case, a massive, relatively small disk forms. This may allow a rapid build-up of planetesimals and protoplanetary cores, but may induce a fast inward migration. In the second example, planetesimals could still be formed in an extended disk on longer timescales. If massive cores were to be formed late in the system their inner migration could be damped. The possibility of forming planetesimals and planets in this variety of scenarios will be studied in a forthcoming paper.

As we will see in the next section, solutions matching the observed surface densities and other constraints are far from being limited to these two cases; a variety of initial conditions and values of the turbulent viscosity can yield satisfactory models. We now turn to an analysis of the ensemble of models that match the observations.

\section{Results: allowed models and derived theoretical constraints}

\subsection{General}

For the most of this section we discuss results on the basis of only 2 parameters representative of the disk physics $(\alpha$ or $\beta$ ) and of the initial conditions $\left(j_{\mathrm{cd}}\right)$, respectively. We thus derive

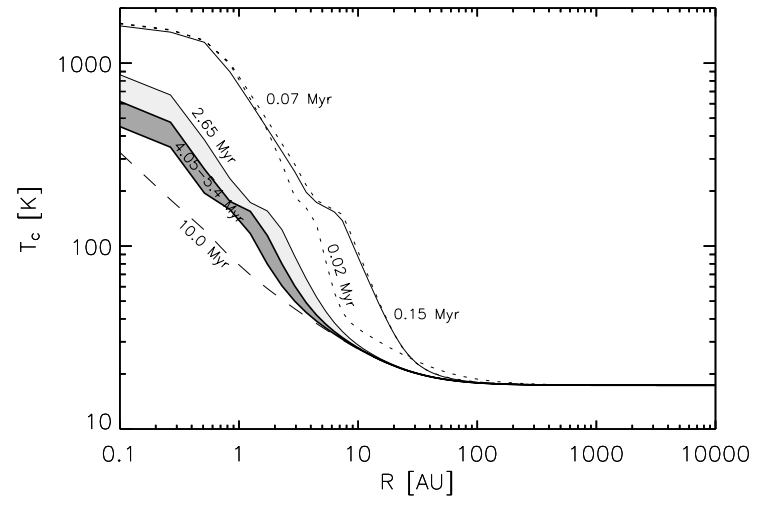

Fig. 10. Example 2. Midplane temperature versus orbital distance. See Fig. 7 and Table 4 for details.

constraints on these parameters depending on the chosen set of observational constraints. Since given values of ( $\alpha$ or $\left.\beta, j_{\mathrm{cd}}\right)$ may be obtained from different combinations of the initial conditions, we also plot the fraction of models that fit the observations. We further checked that the global results are not dependent on the value of $M_{0}$ or on the numerical resolution.

Figure 11 constitutes a summary of our global results for DM Tau and GM Aur for both parameterizations of turbulence. Results are shown for three sets of observational constraints $(\{3\},\{4\}$ and $\{5\})$. The most extended light-grey contours correspond to the envelope in the $\left(v, j_{\mathrm{cd}}\right)$ space covered by models matching the $\mathrm{CO}$ and dust observations, and the star accretion rate with the large error bar (set $\{3\}$ ). This results in relatively weak constraints on the physical parameters. Less restrictive observational constraints are therefore not shown: for DM Tau $\alpha$ models, $30 \%$ of all launched models satisfy the $\mathrm{CO}$ data alone (set $\{1\}$ ) and $18 \%$ satisfy the $\mathrm{CO}+$ dust data (set $\{2\}$ ). If we include the accretion rate information we are able to slightly reduce the number of fitting models to $15 \%$ (set $\{3\}$ ). These constraints are quite unrestrictive because they do not limit the outer radius of the disk and unreasonably massive and extended disks can result.

Adding a reasonable maximum value for the density of the disks at their outer edge (set $\{4\}$ ) yields a significant reduction of the permitted $\left(v, j_{\mathrm{cd}}\right)$ space, either for the DM Tau $\beta$ model or for the GM Aur $\alpha$ and $\beta$ models. This is shown as a dashed contour and grey color in Fig. 11. A still narrower set of solutions can be achieved by restricting the range of allowed accretion rates (set $\{5\}$ ). The final ensemble of solutions is plotted as a thick colored contour in Fig. 11. We consider that this set of solutions provides the most realistic constraints on $v$ and $j_{\mathrm{cd}}$ for DM Tau and GM Aur.

Figure 11 also shows that a right value of $\alpha, \beta$ and $j_{\text {cd }}$ is not a sufficient condition to match the observations. The presence of other initial parameters imply that only a fraction of the models really fit the imposed conditions. This fraction peaks to $60 \%$ for the central region in the DM Tau $\alpha$ panel, $40 \%$ for the $\beta$ case and $50 \%$ and $30 \%$ for GM Aur $\alpha$ and $\beta$ respectively.

The detailed values of the space of parameters allowed for sets $\{2\}$ to $\{5\}$ are given on Table 5. We list the maximum, minimum and statistic mean value of the model parameters that fit each set of conditions. 
Table 5. Summary of the Monte-Carlo exploration for different sets of constraints. The minimum, maximum and mean value of the successful fitting models are given. Symbols: AT Accretion time or molecular cloud collapse time. Units: $j_{\mathrm{cd}}$ in $\mathrm{cm}^{2} \mathrm{~s}^{-1}, R_{\mathrm{c}}$ in AU, $\omega_{\mathrm{cd}}$ in units of $10^{-14} \mathrm{~s}^{-1}$, $T_{\mathrm{cd}}$ in $\mathrm{K}, M_{\mathrm{cd}}$ and $M_{\text {disk }}$ in solar masses, $J_{\mathrm{cd}}$ in $\mathrm{g} \mathrm{cm}^{2} \mathrm{~s}^{-1}$, and AT, Age and $\Delta$ Age in Myr.

\begin{tabular}{|c|c|c|c|c|c|c|c|c|c|c|c|c|c|}
\hline \multirow[t]{3}{*}{ System } & \multirow[t]{3}{*}{ Param. } & \multicolumn{12}{|c|}{ Model results obtained for the different observational constraints } \\
\hline & & \multicolumn{2}{|c|}{ \{2\}: CO + Dust } & $\multicolumn{3}{|c|}{3}:\{2\}+\dot{M}$ & $\multicolumn{4}{|c|}{\mathbf{4}}:\{3\}+$ Outer Limit & $\multicolumn{3}{|c|}{\mathbf{5}}:\{4\}+$ Accurate $\dot{M}$ \\
\hline & & Min & Mean & Max & Min & Mean & Max & Min & Mean & Max & Min & Mean & Max \\
\hline \multirow[t]{12}{*}{ DM Tau } & $\alpha$ & $5 \times 10^{-4}$ & 0.03 & 0.6 & $5 \times 10^{-4}$ & 0.02 & 0.15 & $5 \times 10^{-4}$ & 0.02 & 0.15 & $8 \times 10^{-4}$ & 0.02 & 0.08 \\
\hline & $j_{\mathrm{cd}}$ & 19.0 & 20.1 & 20.8 & 19.2 & 20.1 & 20.7 & 19.2 & 20.0 & 20.7 & 19.3 & 20.0 & 20.7 \\
\hline & $R_{\mathrm{c}}$ & 2.4 & 1090 & 4900 & 4.6 & 980 & 4900 & 4.6 & 720 & 4900 & 7.3 & 750 & 4900 \\
\hline & $\omega_{\mathrm{cd}}$ & 0.14 & 22 & 218 & 0.14 & 23 & 218 & 0.14 & 22 & 218 & 0.14 & 23 & 218 \\
\hline & $T_{\mathrm{cd}}$ & 2 & 15 & 30 & 2 & 16 & 30 & 2 & 16 & 30 & 2 & 16 & 30 \\
\hline & $M_{\mathrm{cd}}$ & 0.41 & 0.59 & 1.0 & 0.41 & 0.56 & 0.87 & 0.41 & 0.53 & 0.69 & 0.41 & 0.53 & 0.69 \\
\hline & $J_{\mathrm{cd}}$ & 52.0 & 53.5 & 54.1 & 52.2 & 53.1 & 54.0 & 52.2 & 53.0 & 53.9 & 52.3 & 53.0 & 53.7 \\
\hline & AT & 0.05 & 0.5 & 4.4 & 0.05 & 0.4 & 4.0 & 0.05 & 0.3 & 4.0 & 0.05 & 0.3 & 3.7 \\
\hline & Age & 1.5 & 3.7 & 7.0 & 1.5 & 3.9 & 7.0 & 1.5 & 3.5 & 7.0 & 1.5 & 3.5 & 6.8 \\
\hline & $\Delta$ Age & 0.05 & 2.6 & 5.5 & 0.05 & 2.3 & 5.5 & 0.05 & 2.0 & 5.5 & 0.05 & 1.0 & 2.8 \\
\hline & $M_{\mathrm{disk}}$ & 0.006 & 0.05 & 0.42 & 0.009 & 0.036 & 0.38 & 0.009 & 0.025 & 0.10 & 0.014 & 0.036 & 0.097 \\
\hline & $N_{\text {models }}$ & & 371 & & & 313 & & & 225 & & & 166 & \\
\hline \multirow[t]{12}{*}{ DM Tau } & $\beta \times 10^{5}$ & 1.1 & 31 & 390 & 1.1 & 24 & 160 & 1.1 & 8.5 & 50 & 2.2 & 8.5 & 50 \\
\hline & $j_{\mathrm{cd}}$ & 19.1 & 20.0 & 20.8 & 19.3 & 20.1 & 20.8 & 19.3 & 19.7 & 20.1 & 19.4 & 19.7 & 20.0 \\
\hline & $R_{\mathrm{c}}$ & 5 & 752 & 4800 & 9 & 810 & 4800 & 9 & 74 & 328 & 15 & 71 & 170 \\
\hline & $\omega_{\mathrm{cd}}$ & 0.05 & 14 & 140 & 0.1 & 15 & 140 & 0.1 & 8 & 36 & 0.1 & 8 & 36 \\
\hline & $T_{\mathrm{cd}}$ & 2 & 14 & 30 & 2 & 15 & 30 & 2 & 15 & 30 & 2 & 15 & 30 \\
\hline & $M_{\mathrm{cd}}$ & 0.413 & 0.65 & 0.97 & 0.42 & 0.65 & 0.97 & 0.42 & 0.56 & 0.72 & 0.43 & 0.55 & 0.65 \\
\hline & $J_{\mathrm{cd}}$ & 52.1 & 53.1 & 54.1 & 52.4 & 53.2 & 54.1 & 52.4 & 52.8 & 53.1 & 52.5 & 52.8 & 53.1 \\
\hline & $\mathrm{AT}$ & 0.06 & 0.7 & 4.9 & 0.06 & 0.6 & 4.9 & 0.06 & 0.4 & 2.6 & 0.06 & 0.5 & 2.6 \\
\hline & Age & 1.5 & 4.0 & 7.0 & 1.5 & 4.0 & 7.0 & 1.5 & 3.2 & 7.0 & 1.5 & 2.7 & 4.5 \\
\hline & $\Delta$ Age & 0.05 & 2.7 & 5.5 & 0.05 & 2.0 & 5.5 & 0.05 & 0.8 & 3.4 & 0.05 & 0.4 & 1.3 \\
\hline & $M_{\mathrm{disk}}$ & 0.01 & 0.15 & 0.57 & 0.01 & 0.20 & 0.50 & 0.01 & 0.06 & 0.13 & 0.02 & 0.07 & 0.13 \\
\hline & $N_{\text {models }}$ & & 277 & & & 235 & & & 75 & & & 44 & \\
\hline \multirow[t]{12}{*}{ GM Aur } & $\alpha$ & $2 \times 10^{-5}$ & 0.02 & 0.3 & $2 \times 10^{-5}$ & 0.005 & 0.03 & $1 \times 10^{-4}$ & 0.003 & 0.02 & $4 \times 10^{-4}$ & 0.002 & 0.01 \\
\hline & $j_{\mathrm{cd}}$ & 19.2 & 20.2 & 20.9 & 19.2 & 20.2 & 20.9 & 19.2 & 19.6 & 20.1 & 19.2 & 19.6 & 20.1 \\
\hline & $R_{\mathrm{c}}$ & 4.1 & 1000 & 4900 & 4.1 & 915 & 4900 & 4.1 & 45 & 233 & 4.1 & 36 & 199 \\
\hline & $\omega_{\mathrm{cd}}$ & 0.04 & 12 & 125 & 0.04 & 11 & 125 & 0.04 & 3.7 & 16 & 0.04 & 3.6 & 12 \\
\hline & $T_{\mathrm{cd}}$ & 2 & 16 & 30 & 2 & 16 & 30 & 2 & 17 & 30 & 2 & 17 & 30 \\
\hline & $M_{\mathrm{cd}}$ & 0.55 & 1.0 & 1.5 & 0.55 & 1.0 & 1.5 & 0.55 & 0.8 & 1.3 & 0.55 & 0.8 & 1.1 \\
\hline & $J_{\mathrm{cd}}$ & 52.3 & 53.5 & 54.4 & 52.3 & 53.5 & 54.4 & 52.3 & 52.9 & 53.4 & 52.3 & 52.8 & 53.3 \\
\hline & AT & 0.07 & 0.6 & 4.9 & 0.07 & 0.6 & 4.7 & 0.07 & 0.4 & 3.7 & 0.07 & 0.5 & 3.7 \\
\hline & Age & 1.0 & 4.5 & 10.0 & 1.0 & 5.5 & 10.0 & 1.0 & 3.4 & 9.8 & 1.0 & 3.2 & 8.6 \\
\hline & $\Delta$ Age & 0.05 & 4.8 & 9.0 & 0.05 & 4.3 & 8.9 & 0.05 & 2.4 & 8.8 & 0.05 & 1.5 & 5.4 \\
\hline & $M_{\mathrm{disk}}$ & 0.03 & 0.30 & 0.90 & 0.03 & 0.25 & 0.80 & 0.03 & 0.11 & 0.34 & 0.03 & 0.10 & 0.28 \\
\hline & $N_{\text {models }}$ & & 785 & & & 563 & & & 159 & & & 88 & \\
\hline \multirow[t]{12}{*}{ GM Aur } & $\beta \times 10^{5}$ & 0.1 & 13 & 180 & 0.1 & 4.8 & 25 & 0.2 & 2.2 & 10 & 0.2 & 1.8 & 8 \\
\hline & $j_{\mathrm{cd}}$ & 19.2 & 20.2 & 20.9 & 19.3 & 20.2 & 20.9 & 19.3 & 19.8 & 20.7 & 19.3 & 19.8 & 20.3 \\
\hline & $R_{\mathrm{c}}$ & 4 & 920 & 4900 & 5 & 930 & 4900 & 5 & 105 & 1900 & 5 & 85 & 500 \\
\hline & $\omega_{\mathrm{cd}}$ & 0.02 & 10 & 120 & 0.02 & 10 & 120 & 0.02 & 4.5 & 30 & 0.1 & 4.3 & 20 \\
\hline & $T_{\mathrm{cd}}$ & 2 & 15 & 30 & 2 & 16 & 30 & 2 & 15 & 30 & 2 & 15 & 30 \\
\hline & $M_{\mathrm{cd}}$ & 0.55 & 1.0 & 1.5 & 0.55 & 1.0 & 1.5 & 0.55 & 0.9 & 1.3 & 0.55 & 0.9 & 1.2 \\
\hline & $J_{\mathrm{cd}}$ & 52.4 & 53.5 & 54.4 & 52.5 & 53.5 & 54.4 & 52.5 & 53.0 & 54.1 & 52.5 & 52.9 & 53.5 \\
\hline & AT & 0.08 & 0.7 & 5 & 0.08 & 0.6 & 5 & 0.08 & 0.6 & 4.8 & 0.08 & 0.5 & 4.3 \\
\hline & Age & 1 & 4.4 & 10 & 1 & 5.1 & 10 & 1 & 4.5 & 10 & 1 & 4.4 & 10 \\
\hline & $\Delta$ Age & 0.05 & 4.9 & 8.9 & 0.05 & 3.8 & 8.9 & 0.05 & 1.6 & 6.1 & 0.05 & 1.0 & 3.9 \\
\hline & $M_{\text {disk }}$ & 0.023 & 0.3 & 0.87 & 0.023 & 0.3 & 0.80 & 0.023 & 0.14 & 0.80 & 0.028 & 0.12 & 0.35 \\
\hline & $N_{\text {models }}$ & & 569 & & & 426 & & & 136 & & & 77 & \\
\hline
\end{tabular}



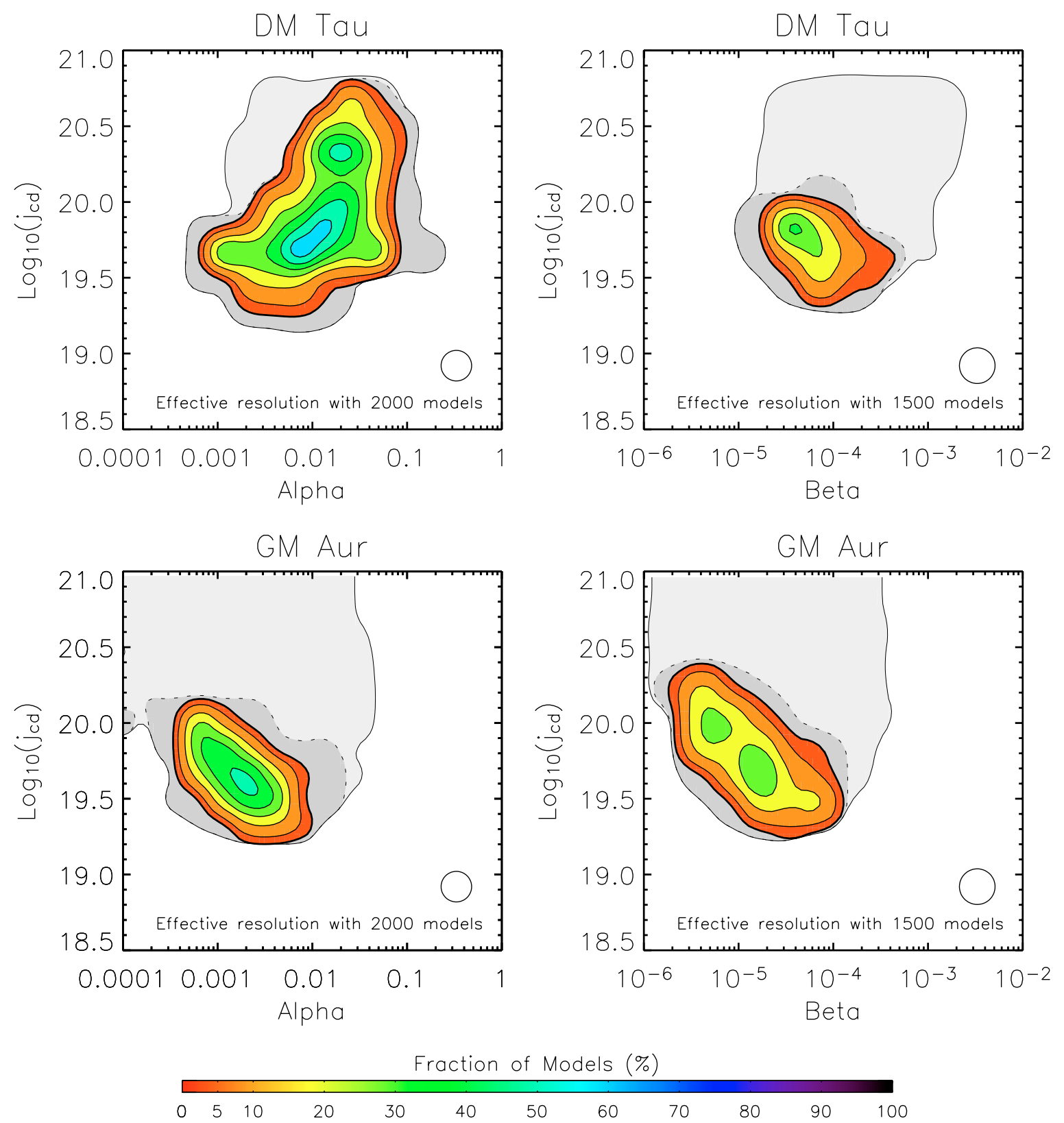

Fig. 11. Viscosity and initial specific angular momentum of models fitting DM Tau and GM Aur for both parameterizations of turbulence. The contour colored plot represents the fraction of models fitting the strongest observational constraints (set $\{5\}$ ). The light-shaded region around, contoured by a dotted line, represents the additional area covered by models assuming an uncertain accretion rate (set $\{4\})$. The light grey filled region surrounding this area corresponds to models with no upper limit on the surface density in the disk's outer region (set $\{3\}$ ).The spatial resolution for each panel is shown as an empty circle. The circle in each figure shows the approximate spatial resolution of the figure obtained by the Monte-Carlo procedure for an accuracy in the fraction of models of $10 \%$. Features in the plots smaller than each circle are not well resolved.

Our first conclusion is that it is possible to use the estimated current state of observed disks to get information about their formation and evolution using relatively simple models. Globally the constraints on the parameters are not very strong, but they anyway provide useful information and ask for further detailed observations of these objects. For instance, a reduction of the star age uncertainty and/or the star accretion rate error bar would be especially useful. Another important conclusion to be drawn is that both $\alpha$ and $\beta$ models provide a satisfactory evolutionary scenario for the disks around DM Tau and
GM Aur. This does not mean that either of these parameterizations are correct but that none of them can be excluded on the basis of these results.

The current disks around DM Tau and GM Aur can be obtained from very different values of the angular momentum in the molecular cloud, corresponding to values of the centrifugal radius spanning the entire range (4 to $5000 \mathrm{AU}$ ) allowed by our numerical approach. A very small value of $R_{\mathrm{c}}$ requires large values of the viscosity to yield an efficient outward diffusion of the disk to match its outer radius. A very large $R_{\mathrm{c}}$ 
also requires a large viscosity, but this time so as to yield a large enough accretion rate. As expected, no useful constraint on $T_{\text {cd }}$ can be derived. Constraints on the initial rotation of the disk $\left(j_{\mathrm{cd}}\right.$ or $\left.\omega_{\mathrm{cd}}\right)$ are weak although models with relatively low values of $j_{\text {cd }}$ seem to be favored.

The mean derived value for the model parameters together with the minimum and maximum allowed values are given on Table 5. The mean value of $\alpha$ inferred from this study is 0.02 for DM Tau and 0.002 for GM Aur, both close to the standard value of $\alpha=0.01$ generally found in the literature (Hartmann et al. 1998). In the $\beta$ parameterization of turbulence the mean values obtained are $9 \times 10^{-5}$ and $2 \times 10^{-5}$ for DM Tau and GM Aur respectively. The $\beta$ value for GM Aur agrees with the prediction that $\beta \approx 2 \times 10^{-5}$ from Richard \& Zahn (1999). The mean value of $\beta$ for DM Tau is a factor of five larger but lower values close to the theoretical prediction are allowed. These conclusions apply to set $\{5\}$, but remain valid for sets $\{2\}$ to $\{4\}$.

A significant part of all models developed gravitational instabilities near the end of the disk-formation era and mostly for a relatively short time span of $1-2 \times 10^{5} \mathrm{yr}$ in systems with ages less than $3 \times 10^{5}$ yr. These thus have a limited impact on the results. We also tested the importance of outflows in the DM Tau $\alpha$ case by arbitarily imposing an inner boundary at $10 \mathrm{AU}$. The extra series of 500 models show no statistically significant difference with the results presented here, except for models with very low values of $j_{\mathrm{cd}}$.

\subsection{Inferred structures of DM Tau and GM Aur}

The selected sets of models provide information not only on the regions where we have observational constraints but also on the general structure of the disks around DM Tau and GM Aur. Figure 12 shows the totality of surface density distributions for the four cases studied and different sets of constraints. The set of models $\{3\}$ and $\{5\}$, are represented as light- and superimposed dark-shaded regions, respectively. Fitting the absolute outer radii of the disks would provide an additional powerful constraint. This may be difficult to obtain since dust observations lack sensitivity and $\mathrm{CO}$ is affected by photodissociation and condensation at low temperatures (Aikawa et al. 1999; Dartois et al. 2003). Unfortunately, at present it is not clear what exactly the outer radii inferred from the millimeter and optical observations imply: they could either be interpreted as an abrupt or as a steady decrease of the density profile. Without assuming a sharp outer edge for GM Aur, models with unreasonable large disk masses become possible fits to the observations (set $\{3\}$ ). On the other hand, sharp outer edges of circumstellar disks would be in better agreement with the $\beta$ prescription of turbulence, as shown by Fig. 12.

Temperature is another important quantity whose evaluation has important implications for planet formation processes. In the $\alpha$ cases it also affects the intensity of the viscous turbulence, $v$, and the disk evolution. The envelopes of all possible radial profiles of temperatures for the fitted models are shown in Fig. 13. These are the thermal profiles of our calculated models at the time when they verify the selected constraints. As in the previous figure, two constraints, $\{3\}$ and $\{5\}$, are shown as light- and dark-shaded regions, respectively. At small radial distances, the temperature is mostly due to viscous dissipation. At large radial distances, the disk becomes optically thin and the temperatures are essentially controlled by the stellar flux. The $\beta$ models are significatively cooler than the $\alpha$ models because they have less mass in the inner disk, implying lower optical depth and lower central temperatures for a given effective (photospheric) temperature. For the same reasons, GM Aur appears to be warmer than DM Tau.

Turbulent viscosity is the key variable to understand the disk evolution. Figure 14 shows the envelope of possible $v$ profiles as a function of stellar distances for the solutions that fit the observations of DM Tau and GM Aur (set $\{5\}$ ). At first glance, the solutions for the $\alpha$ and $\beta$ models may appear to be relatively similar, a consequence of the constraint on the density profile provided by the observations. However, marked departures between the two profiles in the [10-1000 AU] region are apparent. Globally, $v_{\alpha}$ varies as $r^{3 / 4}$, while $v_{\beta}$ varies as $r^{1 / 2}$, meaning than the $\alpha$ models are diffused more efficiently in the outer disk. This explains why, at large distances from the star, the density profile decreases less sharply in the $\alpha$ models than in the $\beta$ models.

\subsection{Global evolution of the disk and gravitational instabilities}

Figure 15 shows the envelope of possible star and disk mass evolution for the models matching conditions $\{3\}$ and $\{5\}$, respectively. Up to $15 \%$ of our selected models (set $\{5\}$ ) were gravitationally unstable, though generally only over a short fraction of their evolution, near the end of the disk formation era. These correspond to the models for which the mass of the disk is close to or exceeds the central star mass. The gravitational instability arises at $30 \pm 10 \mathrm{AU}$ and times $1.0 \pm 0.6 \times 10^{5} \mathrm{yr}$ and typically lasts for $10^{4}-10^{5} \mathrm{yr}$ for DM Tau and less than $2 \times$ $10^{5}$ for GM Aur due to the larger amount of mass the disk has to process. Because we compare observations and models at ages larger than $10^{6} \mathrm{yr}$, gravitational instabilities should not affect signficantly our analysis. In sets $\{4\}$ and $\{5\}$, models with $\alpha>0.03$ were never gravitationally unstable. Models with lower values of $\alpha$ had an imposed $\alpha=0.03$ during gravitational instable periods (see Sect. 3.6.3).

The problem of the evolution of the mass of accretion disks as a function of viscosity and initial angular momentum $J_{\text {cd }}$ was also studied by Nakamoto \& Nakagawa (1995). These authors derived a criterion on the viscosity parameter $\alpha$ which guarantees that at any given time, the disk to star mass ratio never exceeds 0.1 :

$\log \alpha \geq-2.0+4.5 \log J_{\mathrm{cd}}-3.9\left(\log J_{\mathrm{cd}}\right)^{2}$,

with $J_{\text {cd }}$ in units of $10^{53} \mathrm{~g} \mathrm{~cm}^{2} \mathrm{~s}^{-1}$. Their study is relevant for systems of $\sim 1 M_{\odot}$ collapsing in $\sim 6 \times 10^{5} \mathrm{yr}$. They considered the parameter range $J_{\mathrm{cd}}=\left[6 \times 10^{52}-10^{53}\right] \mathrm{g} \mathrm{cm}^{2} \mathrm{~s}^{-1}$ and $\alpha=$ $\left[10^{-5}-0.1\right]$.

For our simulations of DM Tau $\alpha$ we plot in Fig. 16, the regions of the parameter space that have lower masses 

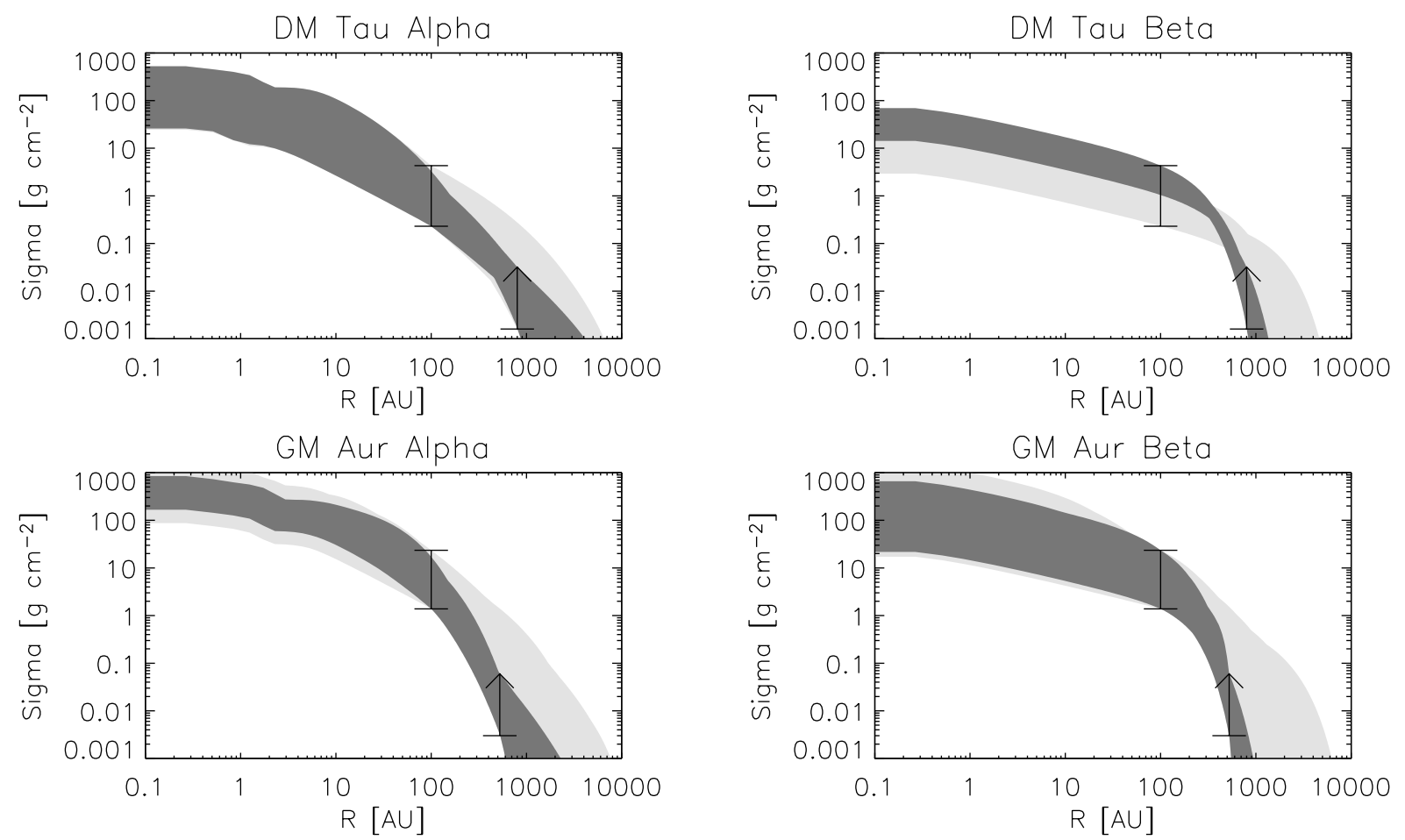

Fig. 12. Radial surface density profile of successful models of DM Tau and GM Aur. The dark-shaded regions correspond to the models fitting the constraints $\{5\}$. The light-shaded regions represent the additional solutions when the constraints are only the CO + dust + Accretion rate with large error bar (set $\{3\}$ ). The error bars are the constraints on $\Sigma$ imposed by $\{5\}$.

than $0.1 M_{*}$ at the end of the disk formation period (and hence, at the moment of maximum disk mass). Our results compare well with those of Nakamoto and Nakagawa, though their result is strictly valid for systems collapsing in 0.6 Myr years and ours extend to systems forming in 0.05 to $5 \mathrm{Myr}$. A comparison with Fig. 11 shows that most solutions for DM Tau and GM Aur are to the left of the critical line, i.e. the disk to star mass ratio has been, or in some cases is still, larger than 0.1 . This seems to contradict the fact that most observed disks appear to have values of this ratio smaller than 0.1 (e.g. Beckwith et al. 1990; Kitamura et al. 2002). However, this contradiction may only be apparent, given the fact that the dust absorption coefficient at millimeter wavelengths may have been overestimated (see Sect. 2.3), and/or that the time spent with a large disk mass is generally relatively short. It is also possible that DM Tau and GM Aur are, among protoplanetary disks, exceptions, and that most other disks have suffered more from either tidal interactions with nearby stars and/or photoevaporation from external, relatively massive stars. Alternatively, our assumptions regarding the uncertain opacity factor may be too conservative and allow for unrealistically large values of the disk mass. This would imply that stronger constraints can be derived from the observations presently available.

\subsection{Results for the collapse of magnetized molecular clouds}

In order to assess the importance of the collapse model in the final results, we also study an $\alpha$ model of DM Tau in which the molecular cloud is assumed to collapse under the influence of a strong magnetic field. Figure 17 shows the final characteristics of models fitting the observational constraints when Eqs. (9), (10) are used. Table 6 shows a summary of our results when fitting the observational constraints $\{2\}$ to $\{5\}$.

Compared with the hydrodynamical collapse models, these are characterized by lower values of the initial rotational velocity $\left(6 \times 10^{-14} \mathrm{~s}^{-1}\right.$ vs. $\left.23 \times 10^{-14} \mathrm{~s}^{-1}\right)$ and similar values of the specific angular momentum $\left(\log _{10}\left(j_{\text {cd }}\right)=20.3\right.$ vs. 20.0$)$. This is because differential rotation yields a faster rotation of the inner molecular cloud core. Furthermore, the final centrifugal radii of the disks are smaller than in the non-magnetic case for a given rotational velocity. The only possible scenario for the formation of DM Tau is an inner disk of intermediate size $\left(R_{\mathrm{c}} \sim 50 \mathrm{AU}\right)$ that expands in time while it diffuses. This is true even if one considers only the weak constraints $\{2\}$.

The distribution of surface densities for the successfully fitting models is shown in Fig. 18. Appart from the fact that high values of the initial angular moment in the cloud core are allowed, the results are similar to those obtained for the nonmagnetic case (Fig. 13), confirming that a strong, sustained viscous evolution of the system is required to fit the observations. In particular, the values of $\alpha$ derived are almost identical regardless of the collapse scenario.

The global evolution of the star and disk masses is shown in Fig. 19. Compared to the non-magnetic case (Fig. 15), the 10 times faster accretion implies that the disks grow very rapidly and are likely to become gravitationally unstable early on. 

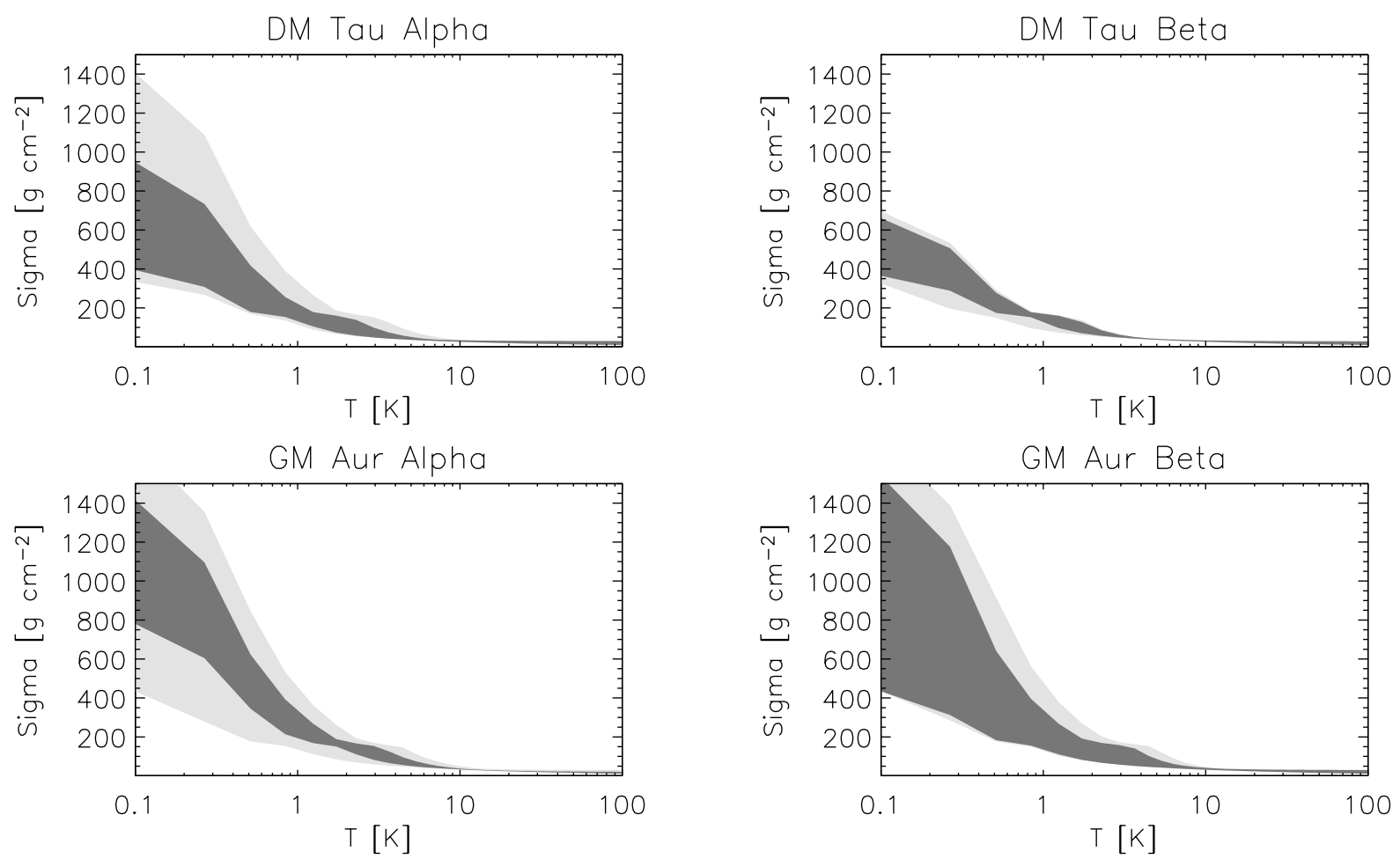

Fig. 13. Ensemble of possible mid-plane disk temperatures for two different sets of observational constraints: $\{3\}$, light-shaded and $\{5\}$, dark-shaded.
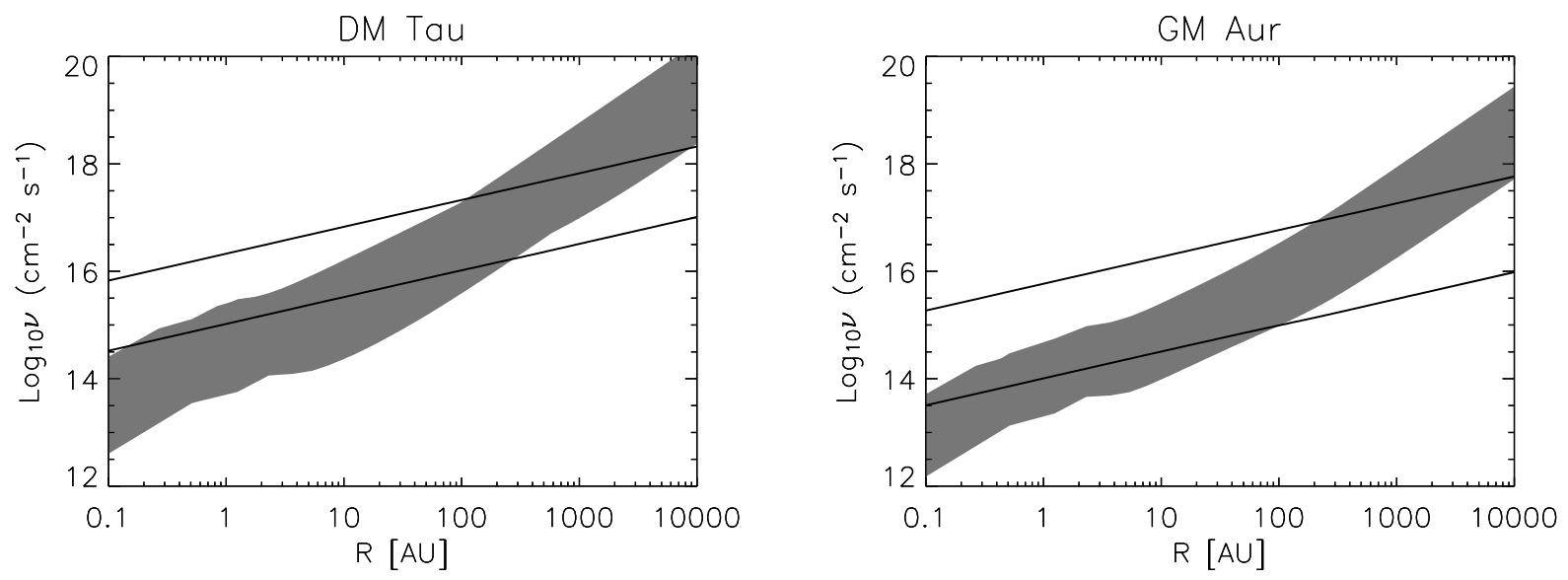

Fig. 14. Viscosities of successful models of DM Tau and GM Aur. Shadowed regions correspond to models calculated with the $\alpha$ parameterization. Lines represent the upper and bottom limit of viscosities for the $\beta$ cases.

\subsection{Turbulence driven by vertical convection?}

One of the advantages of our Monte-Carlo approach is that we can test if some of the different theoretical mechanisms able to produce turbulence in the disk can explain the observations of extended disks. Here we test if the simplest characteristics of thermal convection (Lin \& Papaloizou 1980) can explain the current state of DM Tau. If thermal convection is the dominant source of turbulence in circumstellar disks, the instability should be essentially limited to the inner optically thick disk with a more or less sharp transition in the outer optically thin disk (Ruden \& Pollack 1991).

It is useful to consider some order of magnitude figures. In the outer region the opacities are dominated by ice grains.
For typical temperatures $T \sim 10 \mathrm{~K}$, the Rosseland opacity (gas+dust) is $\kappa_{\mathrm{R}} \sim 0.02 \mathrm{~cm}^{2} \mathrm{~g}^{-1}$ (Pollack et al. 1986). The disk is unable to sustain a convective vertical transport of heat at an optical depth $\tau \lesssim 1$ implying a critical surface density $\Sigma_{\text {crit }} \sim 2 / \kappa_{\mathrm{R}} \sim 100 \mathrm{~g} \mathrm{~cm}^{-2}$. A circumstellar disk of mass $0.1 M_{\odot}$ could then be convective up to a maximum limit of $\sim 50 \mathrm{AU}$. If we consider higher temperatures and disk masses $(T=30 \mathrm{~K}$ and $M_{\mathrm{d}}=0.3 M_{\odot}$ ) this limit extends to $280 \mathrm{AU}$. Therefore, it would be extremely difficult to produce the extended structures of disks like DM Tau and GM Aur by the outward diffusion of initially smaller disks. If turbulent convection is the dominant source of viscosity, these systems should have been formed essentially as they are observed now. 

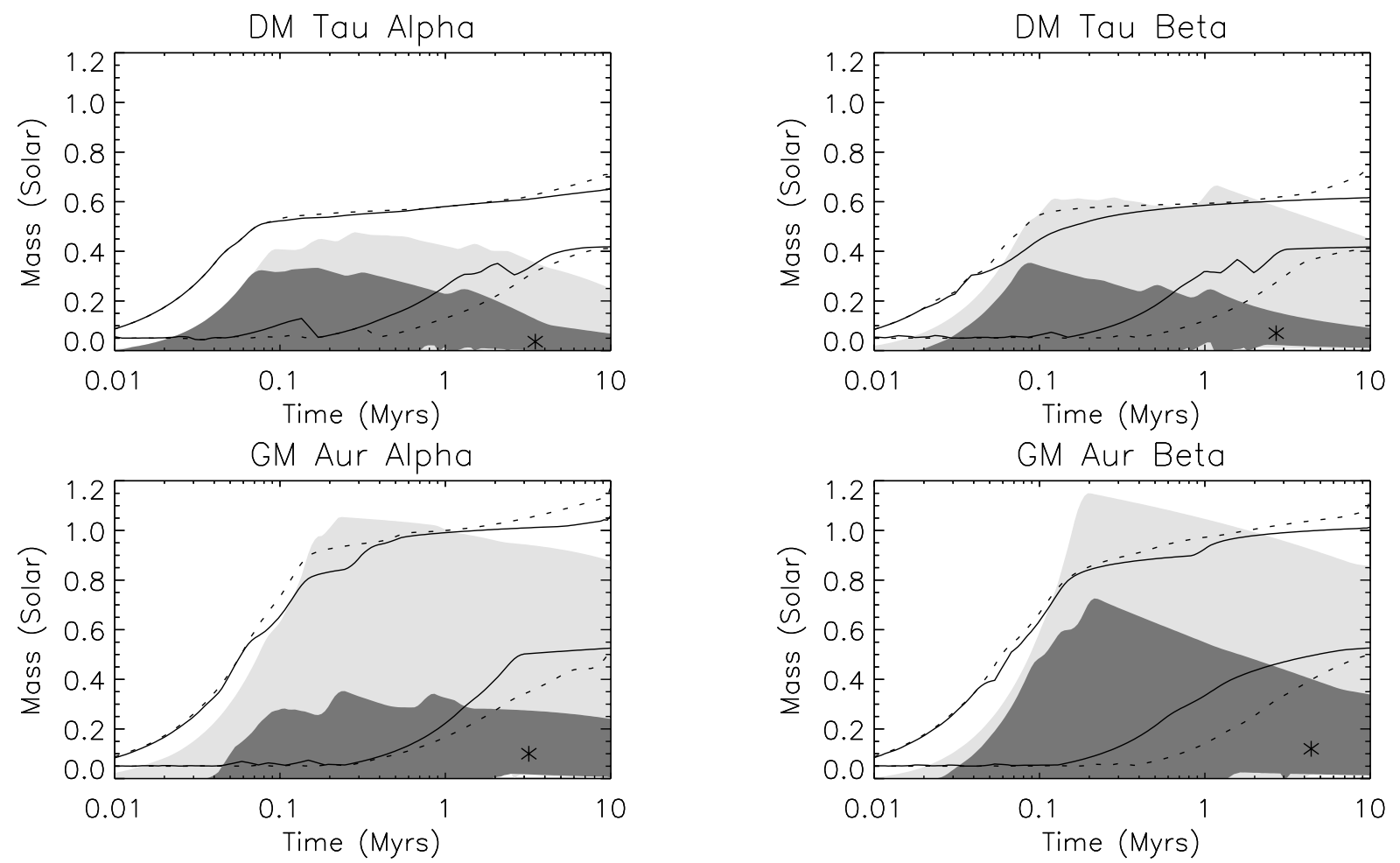

Fig. 15. Star mass and disk mass as a function of time for models succefully fitting selected observational contraints. The light- and dark-shaded regions show the evolution of the disk mass with constraints $\{3\}$ and $\{5\}$, respectively. The dotted and plain lines represent the envelopes of possible star masses with the same constraints. The star symbols mark the mean values of the ages and disk masses of models fulfilling set $\{5\}$.

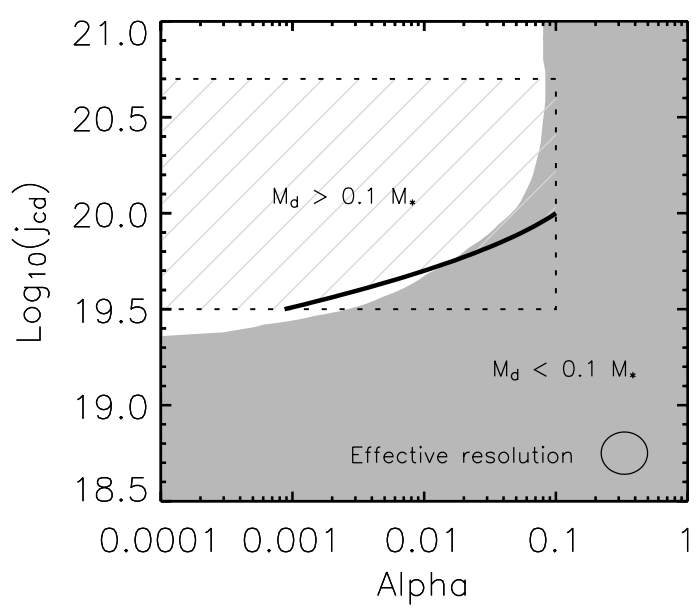

Fig. 16. Regions of the parameter space where massive disk and nonmassive disks can be found. The constraint given by Nakamoto and Nakagawa traces a region (dashed) where massive disks at the end of the molecular cloud collapse are to be found. The dotted box is the area of the space of parameters covered by their study. The grey shaded area represents the region where we found models with disks less massive than $0.1 M_{*}$ at the end of the collapse period. The spatial resolution of our calculations is shown by the lower right circle.

In order to test this possibility we launched a new set of calculations for DM Tau following the same range of values as in the previous cases and considering an $\alpha$ constant in the inner

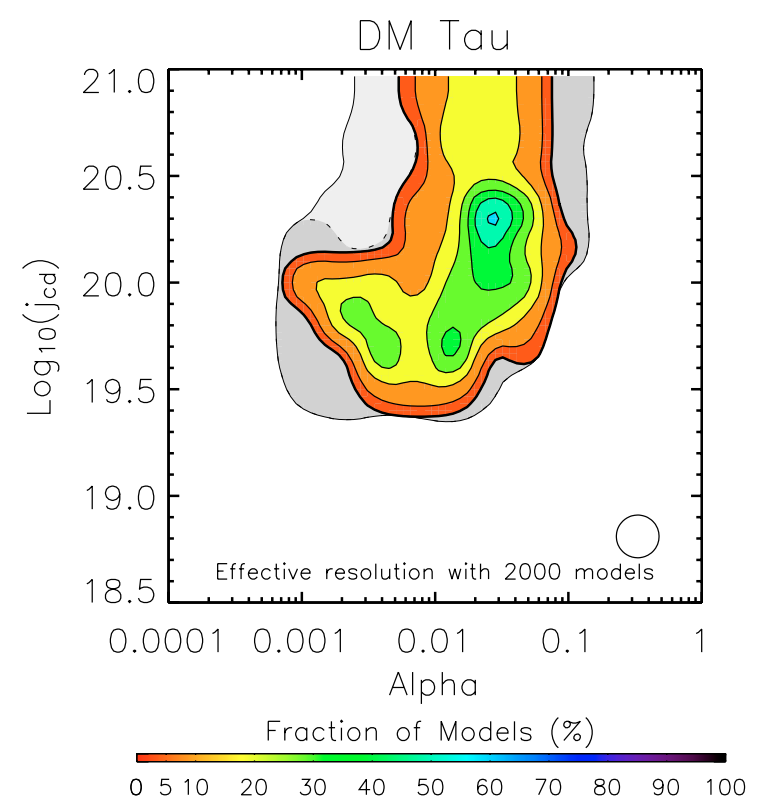

Fig. 17. Distributions of models fitting the sets of constraints $\{3\},\{4\}$ and $\{5\}$ for DM Tau $\alpha$ in the case of an initially magnetized molecular cloud. See Fig. 11 for details.

disk but decreasing in the outer part of the disk as a function of the optical depth to a given power:

$\alpha=\alpha_{0}\left(\frac{\tau}{\tau_{\text {crit }}}\right)^{n} ; \quad \tau \leq \tau_{\text {crit }}$ 
Table 6. Summary of Monte-Carlo exploration in the case of an initial collapse of a magnetized molecular cloud core. For each set of constraints described in the text $\{2\},\{3\}\{4\}$ and $\{5\}$ the minimum, maximum and mean value of the successful fitting models are given. Symbols: AT Accretion time or molecular cloud collapse time. Unities: $j_{\mathrm{cd}}$ in $\mathrm{cm}^{2} \mathrm{~s}^{-1}, R_{\mathrm{c}}$ in $\mathrm{AU}, \omega_{\mathrm{cd}}$ in units of $10^{-14} \mathrm{~s}^{-1}, T_{\mathrm{cd}}$ in $\mathrm{K}, M_{\mathrm{cd}}$ and $M_{\mathrm{disk}}$ in solar masses, $J_{\mathrm{cd}}$ in $\mathrm{g} \mathrm{cm}^{2} \mathrm{~s}^{-1}$, and, AT, Age and $\Delta$ Age in Myr.

\begin{tabular}{|c|c|c|c|c|c|c|c|c|c|c|c|c|c|}
\hline \multirow[t]{3}{*}{ System } & \multirow[t]{3}{*}{ Param. } & \multicolumn{12}{|c|}{ Model results obtained for the different observational constraints } \\
\hline & & $\multicolumn{2}{|c|}{2}: C O+$ Dust & $\multicolumn{3}{|c|}{3}:\{2\}+\dot{M}$ & $\multicolumn{4}{|c|}{4}:\{3\}+$ Outer Limit & $\multicolumn{3}{|c|}{5}:\{4\}+$ Accurate $\dot{M}$ \\
\hline & & Min & Mean & Max & Min & Mean & Max & Min & Mean & Max & Min & Mean & Max \\
\hline \multirow[t]{12}{*}{ DM Tau } & $\alpha$ & $7 \times 10^{-4}$ & 0.03 & 0.2 & $7 \times 10^{-4}$ & 0.02 & 0.1 & $8 \times 10^{-4}$ & 0.03 & 0.1 & 0.004 & 0.02 & 0.1 \\
\hline & $j_{\mathrm{cd}}$ & 19.6 & 20.5 & 21.2 & 19.6 & 20.5 & 21.2 & 19.6 & 20.3 & 21.1 & 19.6 & 20.3 & 21.1 \\
\hline & $R_{\mathrm{c}}$ & 5.4 & 80 & 240 & 5.4 & 70 & 240 & 5.4 & 55 & 200 & 5.4 & 50 & 200 \\
\hline & $\omega_{\mathrm{cd}}$ & 0.7 & 9 & 26 & 0.7 & 8 & 26 & 0.7 & 7 & 26 & 0.7 & 6 & 25 \\
\hline & $T_{\mathrm{cd}}$ & 2 & 14 & 30 & 2 & 13 & 30 & 2 & 15 & 30 & 2 & 15 & 30 \\
\hline & $M_{\mathrm{cd}}$ & 0.41 & 0.60 & 0.97 & 0.41 & 0.58 & 0.86 & 0.41 & 0.54 & 0.67 & 0.42 & 0.54 & 0.67 \\
\hline & $J_{\mathrm{cd}}$ & 52.5 & 53.6 & 54.5 & 52.5 & 53.5 & 54.4 & 52.5 & 53.4 & 54.2 & 52.5 & 53.4 & 54.2 \\
\hline & AT & 0.05 & 0.9 & 9.5 & 0.05 & 0.8 & 9.3 & 0.05 & 0.5 & 9.3 & 0.05 & 0.5 & 9.3 \\
\hline & Age & 1.5 & 3.8 & 7.1 & 1.5 & 4.0 & 7.1 & 1.5 & 3.5 & 7.0 & 1.5 & 3.4 & 7.0 \\
\hline & $\Delta$ Age & 0.05 & 2.3 & 5.6 & 0.05 & 2.1 & 5.6 & 0.05 & 1.7 & 5.5 & 0.05 & 1.0 & 2.7 \\
\hline & $M_{\text {disk }}$ & 0.002 & 0.10 & 0.44 & 0.008 & 0.08 & 0.44 & 0.008 & 0.04 & 0.11 & 0.01 & $\mathbf{0 . 0 3}$ & 0.11 \\
\hline & $N_{\text {models }}$ & & 332 & & & 277 & & & 184 & & & 129 & \\
\hline
\end{tabular}

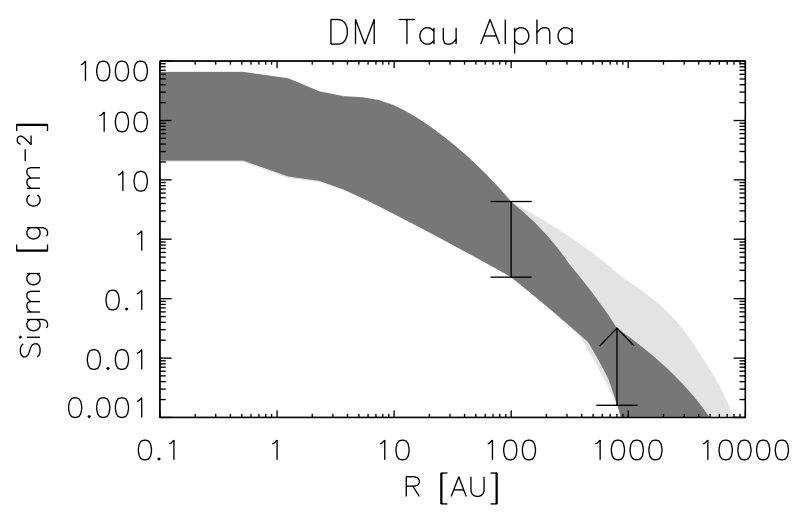

Fig. 18. $\Sigma$ surface density distributions at different times for the successful models in the magnetic cloud case, fulfilling the constraints $\{3\}$ (light-grey) or $\{5\}$ (dark-grey). See Fig. 12 for details.

Here $\alpha_{0}$ characterizes the viscosity in the optically thick disk, $n$ is an integer number that measures the transition of the turbulent active to non active region and $\tau_{\text {crit }}=1.8$ following Ruden \& Pollack (1991). We launched 900 models with $n=8$ (sharp transition) and 500 with $n=1$ (smooth transition) for DM Tau.

In the first case, $n=8$, we obtained 4 models able to simultaneously satisfy the CO and dust constrains (set $\{2\}$ ) with centrifugal radius around $1300 \mathrm{AU}$. These models were typically far too massive and could not satisfy simultaneously the observed accretion rate.

In the second case, we chose $n=1$. This situation corresponds to a slow decrease of turbulence proportional to the optical depth. It is probably unrealistic but could possibly model a hybrid case in which convection is active at $\tau \gtrsim 1$ and another undefined, weaker, turbulent source sets in at lower optical depths. In this case, material can be transported from the optically thin to the optically thick regions.

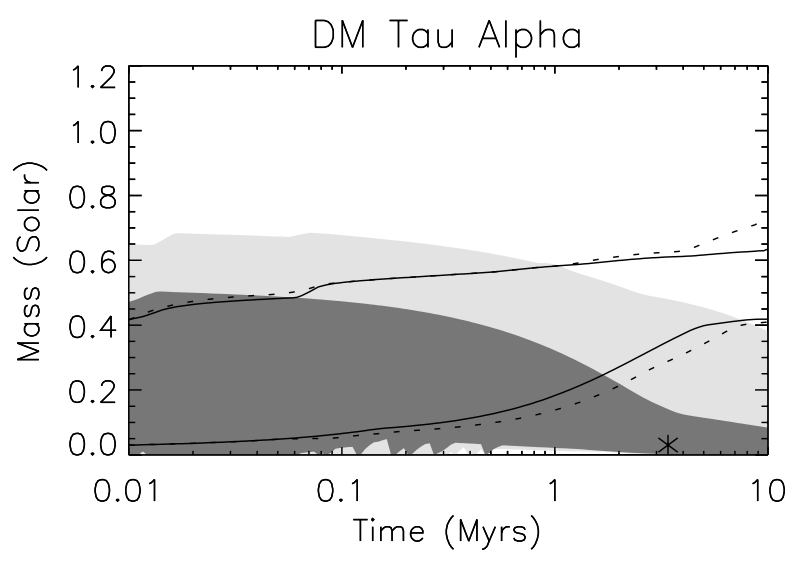

Fig. 19. Star and disk mass evolution for the successful models in the magnetic cloud case. See Fig. 15 for details.

We found that $19 \%$ of all models are able to fit the $\mathrm{CO}+$ dust requirements (set $\{2\}$ ) and that $13 \%$ also fit the accretion rate constraint (set $\{3\}$ ). This is similar to the results obtained previously with a uniform $\alpha$ value. However, the models fitting the constraints $\{3\}$ are now characterized by larger values of the centrifugal radius $\left(R_{\mathrm{c}}=[45,4900] \mathrm{AU}\right)$, specific angular momentum $\left(\log _{10}\left(j_{\mathrm{cd}}\right)=[19.7-20.8]\right)$ and hence viscosity $(\alpha=[0.002,0.6])$ than those listed in Table 5. These disks are generally massive, they develop gravitational instabilities and are formed basically as they are observed. We found that only a tiny fraction $\sim 2 \%$ of all models also fit constraints $\{4\}$. These are characterized by: $R_{\mathrm{c}}=[45,300] \mathrm{AU} ; \alpha=[0.003,0.07]$; $\log _{10}\left(j_{\mathrm{cd}}\right)=[19.7-20.1]$. Finally, only one single model was able to also fit simultaneously the strict accretion rate we have considered on set $\{5\}$.

The global evolution of this model together with the radial distributions of its surface density and the disk effective 


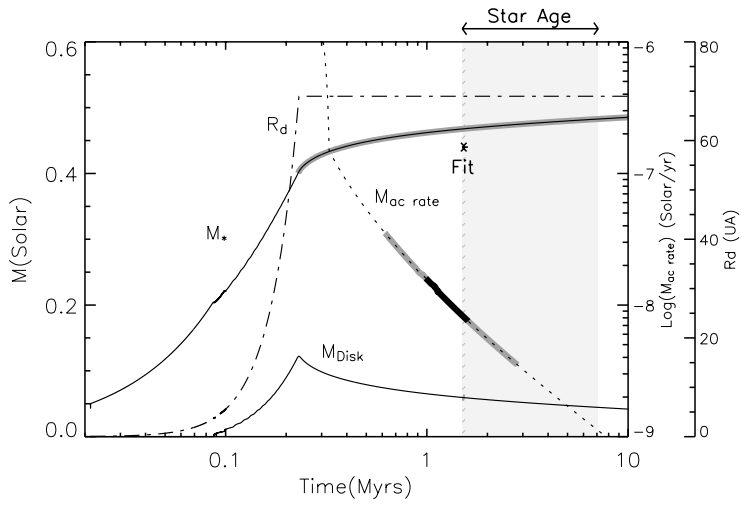

Fig. 20. Global evolution of the star and disk mass. This was the only case found where turbulence of convective origin was found to satisfy the most retrictive set of constraints $\{5\}$. See Fig. 5 for details.

viscosity are shown in Figs. 20-22. The model is characterized by $\alpha=0.07, j_{\mathrm{cd}}=19.73, R_{\mathrm{c}}=62 \mathrm{AU}$ and total accretion time 0.22 Myr. The molecular cloud parameters are also found to agree reasonably well with those inferred for the Taurus formation region $\left(\omega_{\mathrm{cd}}=4.38 \times 10^{-14} \mathrm{~s}^{-1}, T_{\mathrm{cd}}=12 \mathrm{~K}\right.$, $M_{0}=0.05 M_{\odot}$ and $\left.M_{\mathrm{cd}}=0.527 M_{\odot}\right)$. It must be stressed however that this chance result requires initial conditions that are very close to those listed here.

We conclude that it is extremely unlikely that convective instabilities could have been the dominant source of angular momentum transport in DM Tau and GM Aur.

\section{Conclusions}

We presented a (relatively) simple model of evolution of circumstellar disks and applied this model systematically to two well-characterized objects: DM Tau and GM Aur. We chose to survey extensively a rather large parameter space, using various observational constraints from $\mathrm{CO}$ observations of these disks, dust emission properties, derived accretion rates and age and masses of the central stars. Two viscosity parameterizations, $\alpha$ and $\beta$, and two cloud collapse models were used.

We showed that the current state of DM Tau and GM Aur can be understood as resulting from the collapse of a molecular cloud core, the formation of an accretion disk and its spreading due to angular momentum conservation and turbulent diffusive transport. This scenario is consistent with a specific angular momentum initially in the cloud core $\left(j_{\mathrm{cd}} \sim\right.$ $\left.10^{20}-10^{21} \mathrm{~cm}^{2} \mathrm{~s}^{-1}\right)$ similar to measurements in class I protostellar cores (Ohashi et al. 1997), and values of the viscosity that are within an order of magnitude of the standard $\alpha \sim 0.01$ derived from measured ages and accretion rates (e.g. Hartmann et al. 1998). The significant outward diffusion implies that most of the material has been reprocessed and lies far from the position where it had originally fallen onto the disk.

More specifically, the values of $\alpha$ that can be inferred from this study range within $0.001-0.1$ for DM Tau and $4 \times$ $10^{-4}-0.01$ for GM Aur. In the case of the $\beta$ models, the likely values of this parameter are $2 \times 10^{-5}-5 \times 10^{-4}$ for DM Tau and $2 \times 10^{-6}-8 \times 10^{-5}$ for GM Aur.

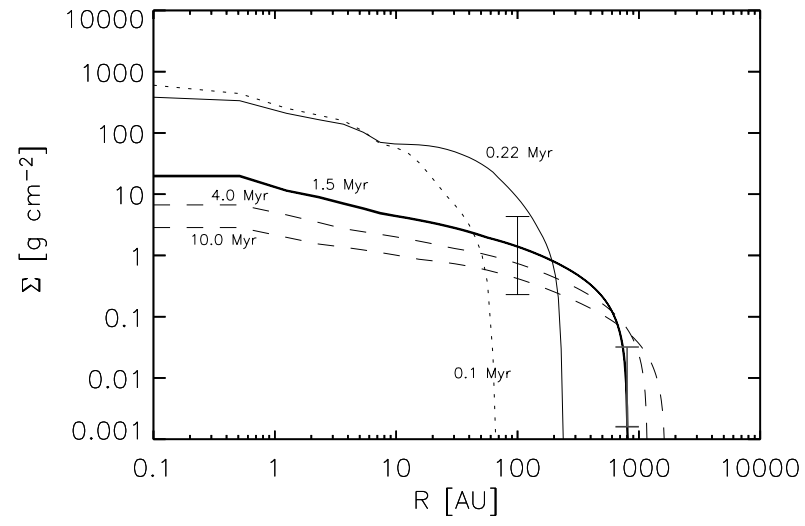

Fig. 21. $\Sigma$ surface density distributions at different times for the same model as the previous figure. See Fig. 6 for details.

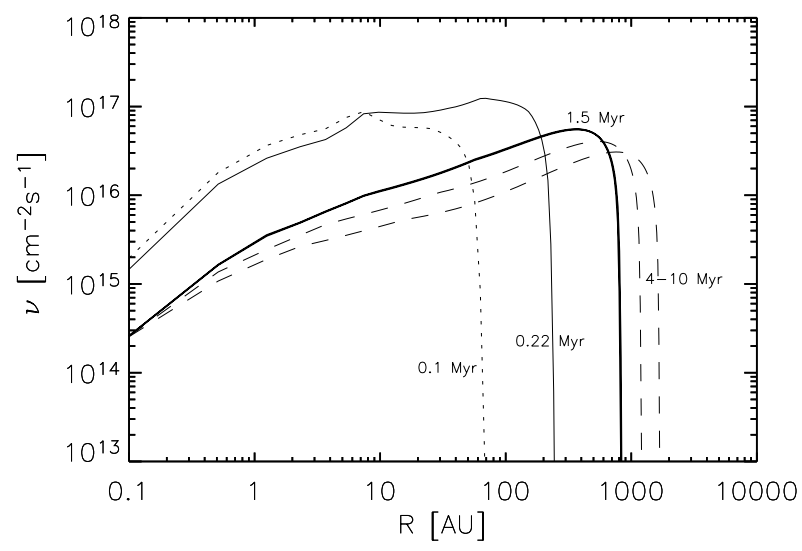

Fig. 22. Radial distribution of turbulent viscosity in the disk as a function of time. As the disk spreads it is able to develop viscosity even in regions far beyond the final centrifugal radius $R_{\mathrm{c}}=62 \mathrm{AU}$. This is because this case corresponds to $n=1$ (see Eq. (30)), implying that the transition between the convective to non-convective regime is extremely smooth (and probably unrealistically so).

Unfortunately, the still relatively large error bars on observationally determined quantities (in particular the age of the system and the star accretion rate) prevent inferring tight constraints on parameters characterizing the systems. We cannot argue in favor of either the $\alpha$ or $\beta$ parameterizations of turbulent viscosity. Similarly, our results are consistent with both a relatively slow (hydrodynamic) or a fast (magnetic) collapse of the primordial molecular cloud core. We can however rule out thermal convection as the main source of turbulence in the disk: the observed disks of DM Tau and GM Aur are too large and extend too far in the optically thin regime in which convection would be suppressed. On the other hand, they cannot be formed directly from the molecular cloud. Convection could still play a role in the inner disk, but another source of turbulence is required in the optically thin regime.

The current state of both systems could be explained by a wide variety of evolutionary histories. In most cases, the disks were always gravitationally stable. However, for low- and highvalues of the initial angular momentum $j_{\mathrm{cd}}$ (corresponding to $R_{\mathrm{c}} \lesssim 10 \mathrm{AU}$ or $\left.R_{\mathrm{c}} \gtrsim 2000 \mathrm{AU}\right)$, disks can become gravitationally unstable for $\sim 10^{5} \mathrm{yr}$ or less. In the case of DM Tau and 
GM Aur, gravitational instabilities thus appear to have had a limited role.

The relatively large values of the viscosity that we derive both for DM Tau and GM Aur imply that an efficient turbulent diffusion mechanism is present throughout these disks. As a consequence, any giant planet forming in these disks will be in risk of migrating rapidly into its star, except if (i) it is protected by an inner dead zone characterized by a low viscosity or (ii) it forms late, i.e. when the local disk mass is comparable to the mass of the planet itself. We regard the second possibility as more likely, but this requires more specific studies. In any case, the presence of extended viscous disks around DM Tau and GM Aur has consequences on how we should apprehend planet formation and should be included in future studies of this problem.

Several improvements to this work are possible. Obviously, our sets of constraints $\{1\}$ to $\{5\}$ should eventually be replaced by a global assessment of the compatibility of the derived density profiles with the different observations (SED, dust emissivity, CO observations, reflected light observations). This is not trivial because of possible variations of the dust to gas ratio (dust migration due to gas drag, condensation at different temperatures), of radial and vertical variations of the size distribution of solid particles, and of variations in the chemical composition (in particular due to $\mathrm{CO}$ condensation and its photodissociation). Another improvement would be to include the thermal evaporation of the disks at large orbital distances (hundreds of AU).

In any case, we believe that meaningful constraints on physical processes leading to the formation of extended disks can now be derived. These critically depend on the inferred surface density profiles at $100 \mathrm{AU}$ and beyond. High sensitivity observations able to detect small amounts of gas or dust in the outer regions of the disks such as forseen for ALMA are of course highly desirable. A better understanding of the inner disk regions and constraints on the rate of accretion onto the star would also be very valuable. Ongoing programs (in particular observations with the Spitzer telescope, but also from ground-based facilities) should ensure a rapid progress towards understanding the formation and evolution of protoplanetary disks.

Acknowledgements. We thank P. Cassen for his comments and suggestions when we took on this project, and A. Dutrey and S. Guilloteau, for providing us with data in advance of publication. The manuscript also benefited from constructive and useful comments from an anonymous referee. We are grateful for discussions with P. André, P. Hennebelle, B. Dubrulle, L. Hartmann, N. Calvet, D. Gautier, P. Michel, and P. Tanga. R. Hueso acknowledges postdoctoral fellowships from Gobierno Vasco and the Observatoire de la Côte d'Azur (bourse Poincaré). This work has been supported by Spanish MCYT research project PNAYA2000-0932, the Universidad del País Vasco Grupos grant 13697/2001 and the French Programme Nationale de Planetologie. Early computations were supported by the OCA program Simulations Interactives et Visualisation en Astronomie et Mécanique (SIVAM). The final calculations were done at the AJAX Cluster at the Grupo de Ciencias Planetarias UPV/EHU.

\section{References}

Adams, F. C., Lada, C. J., \& Shu, F. H. 1988, ApJ, 326, 865

Aikawa, Y., Herbst, E., \& Dzegilenko, F. N. 1999, A\&A, 351, 233

Alexander, R. D., Clarke, C. J., \& Pringle, J. E. 2005, MNRAS, 358, 283

André, P., Ward-Thompson, D., \& Barsony, M. 2000 in Protostars and Planets IV, ed. V. Mannings, A. P. Boss, \& S. S. Russell (Univ. of Arizona Press, Tucson), 59

Balbus, S. A., \& Hawley, J. F. 1991, ApJ, 376, 214

Balbus, S. A., Hawley, J. F., \& Stone, J. M. 1996, ApJ, 467, 76

Balbus, S. A., \& Hawley, J. F. 1998, Rev. Mod. Phys., 70, 1

Balbus, S. A., \& Hawley, J. F. 2000, Space Sci. Rev., 92, 39

Baraffe, I., Chabrier, G., Allard, F., \& Hauschildt, P. H. 1998, A\&A, 337,403

Baraffe, I., Chabrier, G., Allard, F., \& Hauschildt, P. H. 2002, A\&A, 382,563

Basu, S. 1998, ApJ, 509, 229

Bate, M. R., Lubow, S. H., Ogilvie, G. I., \& Miller, K. A. 2003, MNRAS, 341, 213

Bath, G. T., \& Pringle, J. E. 1981, MNRAS, 194, 967

Beckwith, S. V. W., Sargent, A. I., Chini, R. S., \& Guesten, R. 1990, AJ, 99, 924

Bell, K. R., \& Lin, D. N. C. 1994, ApJ, 427, 987

Belloche, A., André, P., Despois, D., \& Blinder, S. 2002, A\&A, 393, 927

Bergin, E., Calvet, N., Sitko, M. L., et al. 2004, ApJ, 614, L133

Bontemps, S., André, P., Terebey, S., \& Cabrit, S. 1996, A\&A, 311, 858

Boss, A. P. 2003, ApJ, 599, 577

Brandenburg, A., Nordlung, A. A., Stein, R. F., \& Torkelsson, U. 1995, ApJ, 446, 741

Calvet, N. 1997, Herbig-Haro Flows and the Birth of Stars (Kluwer), IAU Symp., 182, 417

Casse, F., \& Ferreira, J. 2000, A\&A, 353, 1115

Cassen, P., \& Moosman, A. 1981, Icarus, 48, 353

Cassen, P. 1994, Icarus, 112, 405

Chiang, E. I., \& Goldreich, P. 1997, ApJ, 490, 378

Chiang, E. I., \& Goldreich, P. 1999, ApJ, 519, 279

Clarke, C. J., \& Pringle, J. E. 1991, MNRAS, 249, 584

Clarke, C. J., Bonnell, I. A., \& Hillenbrand, L. A. 2000, in Protostars and Planets IV, ed. V. Mannings, A. P. Boss, \& S. S. Russell, 151

Clarke, C. J., Gendrin, A., \& Sotomayor, M. 2001, MNRAS, 328, 485

D’Alessio, P., Calvet, N., Hartmann, L., Lizano, S., \& Cantó, J. 1999, ApJ, 527, 893

D’Alessio, P., Calvet, N., Hartmann, L., Lizano, S., \& Cantó, J. 2001, ApJ, 553, 321

D’Angelo, G., Henning, T., \& Kley, W. 2002, A\&A, 379, 992

Dartois, E., Dutrey, A., \& Guilloteau, A. 2003, A\&A, 399, 773

van Dishoeck, E. F., Blake, G. A., Draine, B. T., \& Lunine, J. I. 1993, in Protostars and Planets III, ed. E. H. Levy, \& J. Lunine (Tucson: Univ. Arizona Press), 163

Dubrulle, B. 1993, Icarus, 106, 59

Dutrey, A., Guilloteau, S., Prato, L., et al. 1998, A\&A, 338, L63

Foster, P. N., \& Chevalier, R. A. 1993, ApJ, 416, 303

Gammie, C. F. 1996, ApJ, 457, 355

Goldreich, P., \& Lynden-Bell, D. 1965, MNRAS, 130, 97

Goldreich, P., \& Ward, W. R. 1973, ApJ, 183, 1051

Grady, C., Woodgate, B., Stapelfeldt, K., et al. 2002, American Astronomical Society Meeting, 201, 20.20

Guilloteau, S., \& Dutrey, A. 1994, A\&A, 291, L23

Guilloteau, S., \& Dutrey, A. 1998, A\&A, 339, 467

Gullbring, E., Hartmann, L., Briceno, C., \& Calvet, N. 1998, ApJ, 492, 323 
Handa, T., Miyama, S. M., Yamashita, T., et al. 1995, ApJ, 449, 894

Hartmann, L., \& Kenyon, S. J. 1996, ARA\&A, 34, 207

Hartmann, L., Calvet, N., Gullbring, E., \& D’Alessio, P. 1998, ApJ, 495,385

Hawley, J. F., Balbus, S. A., \& Winters, W. F. 1999, ApJ, 518, 394

Hennebelle, P., Whitworth, A. P., Gladwin, P. P., \& André, Ph. 2003, MNRAS, 340, 870

Hollenbach, D. J., Johnstone, D., Lizano, S., \& Shu, F. 1994, ApJ, 428,654

Hollenbach, D. J., Yorke, H. W., \& Johnstone, D. 2000, in Protostars and Planets IV, ed. V. Mannings, A. P. Boss, \& S. S. Russell, 401

Huré, J. M. 2000, A\&A, 358, 378

Huré, J. M., \& Galliano, F. 2001, A\&A, 366, 359

Huré, J. M., Richard, D., \& Zahn, J. P. 2001, A\&A, 367, 1087

Kenyon, S. J., Dobrzycka, D., \& Hartmann, L. 1994, AJ, 108, 1872

Klahr, H. H., Henning, Th., \& Kley, W. 1999, 514, 325

Klahr, H. H., \& Bodenheimer, P. 2001, Joint European and National Meeting JENAM 2001 of the European Astronomical Society and the Astronomische Gesellschaft at Munich, 38

Kitamura, Y., Momose, M., Yokogawa, S., et al. 2002, ApJ, 581, 357

Koerner, D. W., Sargent, A. I., \& Beckwith, S. V. M. 1993, Icarus, 106,2

Konigl, A. 1989, ApJ, 342, 208

Laughlin, G., \& Różyczka, M. 1996, AJ, 456, 279

Laughlin, G., Korchagin, V., \& Adams, F. C. 1997, ApJ, 477, 410

Laughlin, G., Korchagin, V., \& Adams, F. C. 1998, ApJ, 504, 945

Lin, D. N. C., \& Papaloizou, J. 1980, MNRAS, 191, 37

Lin, D. N. C., \& Papaloizou, J. 1996, ARA\&A, 34, 703

Longaretti, P. Y. 2002, ApJ, 576, 587

Lynden-Bell, D., \& Pringle, J. E. 1974, MNRAS, 168, 603

Malbet, F., \& Bertout, C. 1991, ApJ, 383, 814

Matsuyama, I., Johnstone, D., \& Hartmann, L. 2003, ApJ, 582, 893
Miyake, K., \& Nakagawa, Y. 1993, Icarus, 106, 20

Nakamoto, T., \& Nakagawa, Y. 1994, ApJ, 421, 640

Nakamoto, T., \& Nakagawa, Y. 1995, ApJ, 445, 330

O’Dell, C. R., Wen, Z., \& Hu, X. 1993, ApJ, 410, 696

Ohashi, N., Hayashi, M., Ho, P. T. P., et al. 1997, ApJ, 488, 317

Pacyński, B. 1978, AcA, 28, 91

Papaloizou, J. C. B., \& Nelson, R. P. 2003, MNRAS, 339, 983

Pollack, J. B., McKay, C. P., \& Christofferson, B. M. 1986, Icarus, 64, 471

Pollack, J. B., Hollenbach, D., Beckwith, S., et al. 1994, ApJ, 421, 615

Pringle, J. E. 1981, ARA\&A, 19, 137

Reyes-Ruiz, M., \& Stepinski, T. F. 1995, ApJ, 438, 750

Rice, W. K. M., Wood, K., Armitage, P. J., Whitney, B. A., \& Bjorkman, J. E. 2003, MNRAS, 342, 79

Richard, D., \& Zahn, J. P. 1999, A\&A, 347, 734

Ruden, S. P., \& Lin, D. N. C. 1986, ApJ, 308, 883

Ruden, S. P., \& Pollack, J. B. 1991, ApJ, 375, 740

Saito, M., Kawabe, R., Ishiguro, M., et al. 1995, ApJ, 453, 384

Schneider, G., Wood, K., Silverstone, M. D., et al. 2003, AJ, 125, 1467

Shakura, N. I., \& Sunyaev, R. A. 1973, A\&A, 24, 337

Shu, F. H. 1977, ApJ, 214, 488

Shu, F. H., Johnstone, D., \& Hollenbach, D. 1993, Icarus, 106, 92

Simon, M., Dutrey, A., \& Guilloteau, S. 2001, ApJ, 545, 2001

Stone, J. M., \& Balbus, S. A. 1996, ApJ, 463, 656

Stone, J. M., Gammie, C. F., Balbus, S. A. y, \& Hawley, J. F. 2000, Protostars and Planets IV, University of Arizona Press, ed. V. Mannings, A. P. Boss, \& S. S. Russell, 589

Terquem, C. 2002, Proc. Star Formation and the Physics of Young Stars (EDP Sciences), ed. J. Bouvier, \& J. P. Zahn, 203

Toomre, A. 1964, ApJ, 139, 1217

Wood, K., Koerner, D., Whitney, B., et al. 2002, Am. Astron. Soc. Meet., 200, 71.05 